TRANSACTIONS OF THE

AMERICAN MATHEMATICAL SOCIETY

Volume 360, Number 12, December 2008, Pages 6497-6523

S 0002-9947(08)04592-3

Article electronically published on June 26, 2008

\title{
AN EXTENSION OF QUANTITATIVE NONDIVERGENCE AND APPLICATIONS TO DIOPHANTINE EXPONENTS
}

\author{
DMITRY KLEINBOCK
}

\begin{abstract}
We present a sharpening of nondivergence estimates for unipotent (or more generally polynomial-like) flows on homogeneous spaces. Applied to metric Diophantine approximation, it yields precise formulas for Diophantine exponents of affine subspaces of $\mathbb{R}^{n}$ and their nondegenerate submanifolds.
\end{abstract}

\section{INTRODUCTION}

This paper continues the theme started in 1971 by G. A. Margulis Mr1 when he showed that trajectories of one-parameter unipotent flows on $\mathrm{SL}_{k}(\mathbb{R}) / \mathrm{SL}_{k}(\mathbb{Z})$ are never divergent. This had been earlier conjectured by I. Piatetski-Shapiro, and was used by Margulis for the proof of the Arithmeticity Theorem for nonuniform lattices. A decade later, S. G. Dani Da1-Da2], Da4 modified the method of Margulis, showing that any unipotent orbit returns to big compact subsets with high frequency. The latter statement was part of Dani's proof [Da1] of finiteness of locally finite unipotent-invariant ergodic measures and was in M. Ratner's proof [Rt1 - Rt2] of Raghunathan's topological conjecture.

The next development came in 1998, when a very general explicit estimate for the above frequency in terms of the size of compact sets was given in the paper of Margulis and the author $\mathrm{KM}$. In fact it was done in a bigger generality, namely for a large class of maps from $\mathbb{R}^{d}$ into $\mathrm{SL}_{k}(\mathbb{R})$, which made it possible to derive important applications to metric Diophantine approximation on manifolds.

To state some of the results from that paper, recall that the space

$$
\Omega_{k} \stackrel{\text { def }}{=} \mathrm{SL}_{k}(\mathbb{R}) / \mathrm{SL}_{k}(\mathbb{Z})
$$

can be identified with the space of unimodular lattices in $\mathbb{R}^{k}$ and that $\Omega_{k}=$ $\bigcup_{\varepsilon>0} K_{\varepsilon}$, where the sets

$$
K_{\varepsilon} \stackrel{\text { def }}{=}\left\{\Lambda \in \Omega_{k} \mid\|\mathbf{v}\| \geq \varepsilon \text { for all } \mathbf{v} \in \Lambda \backslash\{0\}\right\}
$$

are compact (Mahler's Compactness Criterion; see e.g. [Rg, Corollary 10.9]). Here $\|\cdot\|$ can be any norm on $\mathbb{R}^{k}$, which we will assume to be Euclidean, and extend to the space of discrete subgroups of $\mathbb{R}^{k}$ by letting $\|\Gamma\|$ be the volume of the quotient space $\Gamma_{\mathbb{R}} / \Gamma$ (here and hereafter $\Gamma_{\mathbb{R}}$ stands for the $\mathbb{R}$-linear span of $\Gamma$ ) if $\Gamma \neq\{0\}$, and 1 otherwise.

Received by the editors December 15, 2006.

2000 Mathematics Subject Classification. Primary 37A17; Secondary 11J83.

This work was supported in part by NSF Grant DMS-0239463.

(C)2008 American Mathematical Society 
Another notion we need to introduce is that of functions $(C, \alpha)$-good on an open subset of $\mathbb{R}^{d}$. We postpone a definition of this property until the next section, noting that, roughly speaking, it can be interpreted as some kind of polynomial-like behavior.

The following is a special case of one of the main theorems from [KM]:

Theorem 0.1. Let $d, k \in \mathbb{N}, C, \alpha>0,0<\rho \leq 1$, and let a ball $B=B\left(\mathbf{x}_{0}, r\right) \subset \mathbb{R}^{d}$ and a map $h: \tilde{B} \stackrel{\text { def }}{=} B\left(\mathbf{x}_{0}, 3^{k} r\right) \rightarrow \mathrm{SL}_{k}(\mathbb{R})$ be given. Assume that the following two conditions hold:

(0.1) for any subgroup $\Gamma \subset \mathbb{Z}^{k}$, the function $\mathbf{x} \mapsto\|h(\mathbf{x}) \Gamma\|$ is $(C, \alpha)$-good on $\tilde{B}$,

and

$$
\text { for any subgroup } \Gamma \subset \mathbb{Z}^{k}, \quad \sup _{\mathbf{x} \in B}\|h(\mathbf{x}) \Gamma\| \geq \rho \text {. }
$$

Then

$$
\forall 0<\varepsilon \leq \rho \quad \lambda\left(\left\{\mathbf{x} \in B \mid h(\mathbf{x}) \mathbb{Z}^{k} \notin K_{\varepsilon}\right\}\right) \leq \operatorname{const}(d, k) \cdot C\left(\frac{\varepsilon}{\rho}\right)^{\alpha} \lambda(B),
$$

where the constant above is explicitly computable and depends only on $d$ and $k$.

Here and hereafter $\lambda$ stands for Lebesgue measure on $\mathbb{R}^{d}$.

Roughly speaking, the informal meaning of this theorem is as follows. Under the presence of condition (0.1), which captures the polynomial-like behavior of the map $h$, one of the following two alternatives holds: either most of the 'orbit' $h(B) \mathbb{Z}^{k}$ is contained in $K_{\varepsilon}$, or there exists $\Gamma \subset \mathbb{Z}^{k}$ which is 'responsible for the whole orbit being far away', namely, such that the covolume of $h(\mathbf{x}) \Gamma$ is uniformly small for all $\mathbf{x} \in B$.

Note that in the papers of Margulis and Dani the function $h$ was of the form $x \mapsto u_{x} g$, where $\left\{u_{x}\right\}$ was a unipotent subgroup of $\mathrm{SL}_{k}(\mathbb{R})$ and $g$ a fixed element of $\mathrm{SL}_{k}(\mathbb{R})$. Another class of important applications of Theorem 0.1 is to Diophantine approximation. There, to study Diophantine properties of almost every vector of the form $\mathbf{f}(\mathbf{x})$, where $\mathbf{f}$ is a map from $\mathbb{R}^{d}$ to $\mathbb{R}^{n}$, one takes $k=n+1$ and considers

$$
h(\mathbf{x})=\left(\begin{array}{cc}
e^{-t} & 0 \\
0 & e^{t / n} I_{n}
\end{array}\right)\left(\begin{array}{cc}
1 & \mathbf{f}(\mathbf{x}) \\
0 & I_{n}
\end{array}\right) .
$$

In this case checking (0.1) amounts to establishing polynomial-like behavior of $\mathbf{f}$, and (0.2) can often be extracted from global Diophantine properties of the image of f. The papers [KM], BKM], BBKM], G1, K2-3], [KLW, [KT, KW1-3] contain various number-theoretic applications of Theorem 0.1 and its generalizations. See also [K1, Mr2 for a survey of this method and related results.

The purpose of the present paper is to pay special attention to condition $(0.2)$ of Theorem 0.1. Namely, it is not surprising that the higher is the $\operatorname{rank} \operatorname{rk}(\Gamma)$ of $\Gamma$ (defined as the rank of $\Gamma$ as a $\mathbb{Z}$-module, or, equivalently, the dimension of $\Gamma_{\mathbb{R}}$ ), the harder is usually the task of estimating $\|h(\cdot) \Gamma\|$ from below. For example if $\Gamma$ is of rank $j$ and $h(\mathbf{x}) \Gamma$ is generated by $j$ orthogonal vectors of length $\rho$, one has $\|h(\mathbf{x}) \Gamma\| \asymp \rho^{j}$. Indeed, in some cases relevant to Diophantine applications one can prove estimates of the following form:

$$
\text { for any } \Gamma \subset \mathbb{Z}^{k}, \quad \sup _{\mathbf{x} \in B}\|h(\mathbf{x}) \Gamma\| \geq \rho^{\mathrm{rk}(\Gamma)} .
$$


This leads to a natural question - whether or not such a condition is enough for deriving (0.3). It became clear to the author in the process of working on the paper K2 that an affirmative answer to the latter question would make it possible to significantly generalize several key results from that paper.

The following theorem provides such an answer:

Theorem 0.2. Let $d, k \in \mathbb{N}, C, \alpha, \rho>0$, and suppose that a ball $B=B\left(\mathbf{x}_{0}, r\right) \subset \mathbb{R}^{d}$ and a map $h: \tilde{B} \stackrel{\text { def }}{=} B\left(\mathbf{x}_{0}, 3^{k} r\right) \rightarrow \mathrm{SL}_{k}(\mathbb{R})$ satisfy $(0.1)$ and $\left(0.2^{\prime}\right)$. Then (0.3) holds.

We will comment on the geometric significance of the improvement of Theorem 0.2 over Theorem 0.1 at the end of $\S 2$, after a more general result is proved.

Let us now describe some number-theoretic applications of the above theorem. For $m, n \in \mathbb{N}, M_{m, n}$ will stand for the space of real matrices with $m$ rows and $n$ columns. Define the Diophantine exponent $\omega(A)$ of $A \in M_{m, n}$ (sometimes called 'the exact order' of $A$ ) to be the supremum of $v>0$ for which there are infinitely many $\mathbf{q} \in \mathbb{Z}^{n}$ such that

$$
\|A \mathbf{q}+\mathbf{p}\|<\|\mathbf{q}\|^{-v}
$$

for some $\mathbf{p} \in \mathbb{Z}^{m}$. Note that this quantity is independent of the choice of norms on $\mathbb{R}^{m}$ and $\mathbb{R}^{n}$ (thus we will repeatedly switch between Euclidean and supremum norms whenever it is convenient). It is well known and easy to see that one has $n / m \leq \omega(A) \leq \infty$ for all $A \in M_{m, n}$, and $\omega(A)=n / m$ for $\lambda$-almost every $A \in M_{m, n}$.

We will specialize to the case $m=1$, that is, consider Diophantine properties of $\mathbf{y} \in \mathbb{R}^{n}$ interpreted as row vectors (see however $\S 6.3$ for the column vector setup). Further, our emphasis will be on Diophantine approximation with dependent quantities, where the dependence is expressed by means of a Borel measure $\mu$ on $\mathbb{R}^{n}$. Namely, let us define the Diophantine exponent $\omega(\mu)$ of $\mu$ to be the $\mu$-essential supremum of the function $\omega(\cdot)$; in other words,

$$
\omega(\mu) \stackrel{\text { def }}{=} \sup \{v \mid \mu(\{\mathbf{y} \mid \omega(\mathbf{y})>v\})>0\} .
$$

Clearly it only depends on the measure class of $\mu$. If $\mu$ is naturally associated with a subset $\mathcal{M}$ of $\mathbb{R}^{n}$ supporting $\mu$ (for example, if $\mathcal{M}$ is a smooth submanifold of $\mathbb{R}^{n}$ and $\mu$ is the measure class of the Riemannian volume on $\mathcal{M} \Longleftrightarrow$ the pushforward $\mathbf{f}_{*} \lambda$ of $\lambda$ by any smooth map $\mathbf{f}$ parametrizing $\mathcal{M}$ ), we will define the Diophantine exponent $\omega(\mathcal{M})$ of $\mathcal{M}$ to be equal to that of $\mu$. Clearly $\omega(\mu) \geq n$ for any $\mu$, and $\omega(\lambda)=\omega\left(\mathbb{R}^{n}\right)$ is equal to $n$. The latter justifies the terminology introduced to Diophantine approximation on manifolds by V. Sprindžuk: a measure $\mu$ on $\mathbb{R}^{n}$ (resp., a submanifold $\mathcal{M}$ of $\mathbb{R}^{n}$ ) is extremal if $\omega(\mu)$ (resp., $\omega(\mathcal{M})$ ) is equal to $n$, that is, attains the smallest possible value.

It was conjectured by Sprindžuk in 1980 Sp and proved in [KM] that real analytic manifolds not contained in any proper affine subspace of $\mathbb{R}^{n}$ are extremal. More generally, let us say that a differentiable map $\mathbf{f}: U \rightarrow \mathbb{R}^{n}$, where $U$ is an open subset of $\mathbb{R}^{d}$, is nondegenerate in an affine subspace $\mathcal{L}$ of $\mathbb{R}^{n}$ at $\mathbf{x} \in U$ if $\mathbf{f}(U) \subset \mathcal{L}$ and the span of all the partial derivatives of $\mathbf{f}$ at $\mathbf{x}$ up to some order coincides with the linear part of $\mathcal{L}$. If $\mathcal{M}$ is a $d$-dimensional submanifold of $\mathcal{L}$, we will say that $\mathcal{M}$ is nondegenerate in $\mathcal{L}$ at $\mathbf{y} \in \mathcal{M}$ if any (equivalently, some) diffeomorphism $\mathbf{f}$ between an open subset $U$ of $\mathbb{R}^{d}$ and a neighborhood of $\mathbf{y}$ in $\mathcal{M}$ is nondegenerate in $\mathcal{L}$ at $\mathbf{f}^{-1}(\mathbf{y})$. We will say that $\mathbf{f}: U \rightarrow \mathcal{L}$ (resp., $\mathcal{M} \subset \mathcal{L}$ ) is nondegenerate in 
$\mathcal{L}$ if it is nondegenerate in $\mathcal{L}$ at $\lambda$-a.e. point of $U$ (resp., of $\mathcal{M}$, in the sense of the smooth measure class on $\mathcal{M}$ ).

The next theorem generalizes some of the results of [KM] and [K2]:

Theorem 0.3. Let $\mathcal{L}$ be an affine subspace of $\mathbb{R}^{n}$, and let $\mathcal{M}$ be a submanifold of $\mathcal{L}$ which is nondegenerate in $\mathcal{L}$. Then

$$
\omega(\mathcal{M})=\omega(\mathcal{L})=\inf \{\omega(\mathbf{y}) \mid \mathbf{y} \in \mathcal{L}\}=\inf \{\omega(\mathbf{y}) \mid \mathbf{y} \in \mathcal{M}\} .
$$

In short, Diophantine exponents of affine subspaces are inherited by their nondegenerate submanifolds. This was proved in $\overline{\mathrm{KM}}$ for $\mathcal{L}=\mathbb{R}^{n}$, and in $\mathrm{K2}$ for extremal $\mathcal{L}$ (that is, with $\omega(\mathcal{L})=n$ ).

Note that the middle equality is trivially satisfied for $\mathcal{L}=\mathbb{R}^{n}$, but is not at all obvious for proper subspaces $\mathcal{L}$. Indeed, it states that the infimum of $\left.\omega\right|_{\mathcal{L}}$ coincides with its essential supremum; that is, the existence of a single point $\mathbf{y} \in \mathcal{L}$ with $\omega(\mathbf{y}) \leq v$ forces the set $\{\mathbf{y} \in \mathcal{L} \mid \omega(\mathbf{y}) \leq v\}$ to have full measure.

Another natural problem addressed in the paper is computing Diophantine exponents of affine subspaces in terms of the coefficients of their parametrizing maps. If $\mathcal{L}$ is an $s$-dimensional affine subspace of $\mathbb{R}^{n}$, by permuting variables one can without loss of generality choose a parametrizing map of the form $\mathbf{x} \mapsto\left(\mathbf{x}, \mathbf{x} A^{\prime}+\mathbf{a}_{0}\right)$, where $A^{\prime} \in M_{s, n-s}$ and $\mathbf{a}_{0} \in \mathbb{R}^{n-s}$ (here both $\mathbf{x}$ and $\mathbf{a}_{0}$ are row vectors). It will be convenient to denote the matrix $\left(\begin{array}{l}\mathbf{a}_{0} \\ A^{\prime}\end{array}\right)$ by $A \in M_{s+1, n-s}$, so that $\mathcal{L}$ is parametrized by

$$
\mathbf{x} \mapsto(\mathbf{x}, \tilde{\mathbf{x}} A)
$$

where $\tilde{\mathbf{x}}$ stands for $(1, \mathbf{x})$.

One of the advantages of such a parametrization is a possibility to relate Diophantine properties of $A$ to those of points of $\mathcal{L}$. Indeed, following [K2, it can be easily shown that a good approximation to $A$ gives rise to a good approximation to all points of $\mathcal{L}$ simultaneously (see Lemma 5.4). Consequently, $\omega(A)$ is a lower bound for $\omega(\mathcal{L})$; thus

$$
\omega(\mathcal{L}) \geq \max (\omega(A), n) .
$$

Estimating $\omega(\mathcal{L})$ from above is a more difficult task. We accomplish it in $\S 5$ by writing a precise expression for $\omega(\mathcal{L})$ parametrized as in $(0.6)$ in terms of $A$ (see Corollary 5.2). In particular, we prove

Theorem 0.4. If a proper affine subspace $\mathcal{L}$ of $\mathbb{R}^{n}$ is parametrized as in (0.6), where either (a) all the columns or (b) all the rows of $A$ are rational multiples of one column (resp., row), then (0.7 $\geq$ ) turns into equality; that is, one has

$$
\omega(\mathcal{L})=\max (\omega(A), n) .
$$

This generalizes [K2, Theorem 1.3]. Examples of subspaces described by Theorem 0.4 include: those parallel to coordinate subspaces; lines passing through the origin; subspaces of codimension one. Whether or not $(0.7=)$ holds in general is an open question; see $\S 6.1-2$ for discussion.

We remark that the main results of this paper, Theorems 2.1 and 2.2, are much more general than Theorem 0.2. Namely, we consider maps from Besicovitch metric spaces equipped with Federer measures; see $\S 1$ for definitions. Thus our main Diophantine result, Theorem 1.3, is substantially more general than Theorem 0.3. 
In particular, its framework includes fractal subsets of $\mathbb{R}^{n}$ or, more generally, measures of the form $\mathbf{f}_{*} \mu$ where $\mu$ satisfies certain decay conditions, as in [KLW]. In $\S 1$ we review all the necessary terminology and background facts, and in $\S 2$ prove the general quantitative nondivergence estimates. Section 3 describes a connection between Diophantine approximation and dynamics which makes it possible to apply Theorem 2.2 to Diophantine exponents. Theorem 1.3 is proved in $\S 4$ and Theorem 0.4 in $\S 5$. In fact, the Diophantine exponents of arbitrary affine subspaces are expressed in terms of so-called higher order Diophantine exponents of matrices, which are introduced and studied in detail in $\S 5$. The last section contains several open questions and further generalizations of the Diophantine problems considered in the paper.

\section{Preliminaries (Besicovitch, Federer, Good, nonplanar) AND THE MAIN DiOPHANTINE RESULTS}

A metric space $X$ is called $N$-Besicovitch if for any bounded subset $A$ of $X$ and any family $\mathcal{B}$ of nonempty open balls in $X$ such that each $x \in A$ is a center of some ball of $\mathcal{B}$, there is a finite or countable subfamily $\left\{B_{i}\right\}$ of $\mathcal{B}$ covering $A$ with multiplicity at most $N$. We will say that $X$ is Besicovitch if it is $N$-Besicovitch for some $N$. The fact that $\mathbb{R}^{d}$ is Besicovitch is the content of Besicovitch's Covering Theorem [Mt, Theorem 2.7].

Let $\mu$ be a Radon measure on $X$, and $U$ an open subset of $X$ with $\mu(U)>0$. Following [KLW], let us say that $\mu$ is $D$-Federer on $U$ if

$$
\sup _{\substack{x \in \operatorname{supp} \mu, r>0 \\ B(x, 3 r) \subset U}} \frac{\mu(B(x, 3 r))}{\mu(B(x, r))}<D .
$$

Equivalently, one can replace ' 3 ' in (1.1) by any $c>1$, appropriately changing the value of $D$. This explains why Federer measures are often called doubling. It will be useful to have a nonuniform version of the above definition: we will say that $\mu$ as above is Federer if for $\mu$-a.e. $x \in X$ there exists a neighborhood $U$ of $x$ and $D>0$ such that $\mu$ is $D$-Federer on $U$. Many natural examples of measures, including those supported on fractals, can be shown to be Federer.

For a subset $B$ of $X$ and a function $f$ from $X$ to a normed space with norm $\|\cdot\|$, we let $\|f\|_{B} \stackrel{\text { def }}{=} \sup _{x \in B}\|f(x)\|$. If $\mu$ is a measure on $X$ and $B$ is a subset of $X$ with $\mu(B)>0$, we define $\|f\|_{\mu, B}$ to be equal to $\|f\|_{B \cap \operatorname{supp} \mu}$.

A function $f: X \rightarrow \mathbb{R}$ is called $(C, \alpha)$-good on $U \subset X$ with respect to $\mu$ if for any open ball $B \subset U$ centered in $\operatorname{supp} \mu$ one has

$$
\forall \varepsilon>0 \quad \mu(\{x \in B|| f(x) \mid<\varepsilon\}) \leq C\left(\frac{\varepsilon}{\|f\|_{\mu, B}}\right)^{\alpha} \mu(B) .
$$

Informally speaking, a function is good if the set of points where it takes small values has small measure. We refer the reader to [KM], $\mathrm{BKM}$, [KLW], $\mathrm{KT}$ ] for various properties and examples. One of the elementary observations is conveniently stated below:

Lemma 1.1 ([KLW, Lemma 4.1]). Suppose that $f_{1}, \ldots, f_{\ell}$ are $(C, \alpha)$-good on $U$ with respect to $\mu$. Then $\left(f_{1}^{2}+\cdots+f_{\ell}^{2}\right)^{1 / 2}$ is $\left(\ell^{\alpha / 2} C, \alpha\right)$-good on $U$ with respect to $\mu$. 
In the situations when $U$ is a subset of $\mathbb{R}^{d}$ and $\mu=\lambda$, we will omit the reference to the measure and will simply say ' $f$ is $(C, \alpha)$-good on $U$ ', as has already been done in (0.1). In that case one can replace $\|f\|_{\mu, B}$ in (1.2) by $\|f\|_{B}$ and not pay attention to the restriction of the center of $B$ lying in the support of the measure.

$(C, \alpha)$-good functions often come in families. For example, condition (0.1) used in Theorems 0.1 and 0.2 asserts that functions of the form $\mathbf{x} \mapsto\|h(\mathbf{x}) \Gamma\|$, where $\Gamma$ runs through subgroups of $\mathbb{Z}^{k}$, are all $(C, \alpha)$-good with uniform $C$ and $\alpha$. Often we will need to check the $(C, \alpha)$-good property for functions from a given finitedimensional function space. An example: polynomials in $d$ variables are $(C, \alpha)$-good on $\mathbb{R}^{d}$ with $C$ and $\alpha$ depending only on $d$ and the degree of the polynomial.

The following definition was introduced in $\left[\mathrm{K} 2\right.$ and $\mathrm{KT}$. Let $\mathbf{f}=\left(f_{1}, \ldots, f_{n}\right)$ be a map from a metric space $X$ to $\mathbb{R}^{n}$ and $\mu$ a measure on $X$. We will say that $(\mathbf{f}, \mu)$ is good at $x \in X$ if there exists a neighborhood $V$ of $x$ and positive $C, \alpha$ such that any linear combination of $1, f_{1}, \ldots, f_{n}$ is $(C, \alpha)$-good on $V$ with respect to $\mu$. We will simply say that $(\mathbf{f}, \mu)$ is good if it is good at $\mu$-almost every point. Again, the reference to the measure will be omitted when $\mu=\lambda$, in which case we will say that $\mathbf{f}$ is good or good at $x$. For example, we will say that polynomial maps are good (in fact, good at every point). More generally, based on the work done in [KM], the following was proved in [K2]:

Lemma 1.2. Let $\mathcal{L}$ be an affine subspace of $\mathbb{R}^{n}$ and let $\mathbf{f}$ be a smooth map from open $U \subset \mathbb{R}^{d}$ to $\mathcal{L}$ which is nondegenerate in $\mathcal{L}$ at $\mathbf{x} \in U$. Then $\mathbf{f}$ is good at $\mathbf{x}$.

For a subset $M$ of $\mathbb{R}^{n}$, define its affine span $\langle M\rangle_{a}$ to be the intersection of all affine subspaces of $\mathbb{R}^{n}$ containing $M$. Then it is easy to see that $\mathcal{L}$ in the above lemma is equal to $\langle\mathbf{f}(B)\rangle_{a}$ for some open $B \ni \mathbf{x}$. It will be useful to define a similar property for more general maps and measures. Namely, let $X$ be a metric space, $\mu$ a measure on $X, \mathcal{L}$ an affine subspace of $\mathbb{R}^{n}$ and $\mathbf{f}$ a map from $X$ into $\mathcal{L}$. Say that $(\mathbf{f}, \mu)$ is nonplanar in $\mathcal{L}$ (cf. [KT, $\S 4]$ and [KW3, $\S 1]$ ) if

$$
\mathcal{L}=\langle\mathbf{f}(B \cap \operatorname{supp} \mu)\rangle_{a} \quad \forall \text { nonempty open } B \text { with } \mu(B)>0 .
$$

As before, we will omit the dependence on $\mu$ (resp., $\mathcal{L})$ when $\mu=\lambda\left(\right.$ resp., $\mathcal{L}=\mathbb{R}^{n}$ ). Clearly $(\mathbf{f}, \mu)$ is nonplanar iff for any nonempty open $B$ of positive measure the restrictions of $1, f_{1}, \ldots, f_{n}$ to $B \cap \operatorname{supp} \mu$ are linearly independent over $\mathbb{R}$.

As was said before, a basic example is given by nondegenerate smooth maps from $\mathbb{R}^{d}$ to $\mathbb{R}^{n}$ : it is clear from the definition that nondegeneracy in $\mathcal{L}$ implies nonplanarity in $\mathcal{L}$. Thus the following statement generalizes Theorem 0.3:

Theorem 1.3. Let $\mu$ be a Federer measure on a Besicovitch metric space $X, \mathcal{L}$ an affine subspace of $\mathbb{R}^{n}$, and let $\mathbf{f}: X \rightarrow \mathcal{L}$ be a continuous map such that $(\mathbf{f}, \mu)$ is good and nonplanar in $\mathcal{L}$. Then

$$
\omega\left(\mathbf{f}_{*} \mu\right)=\omega(\mathcal{L})=\inf \{\omega(\mathbf{y}) \mid \mathbf{y} \in \mathcal{L}\}=\inf \{\omega(\mathbf{f}(x)) \mid x \in \operatorname{supp} \mu\} .
$$

The special case $\mathcal{L}=\mathbb{R}^{n}$ was (in a slightly different terminology) one of the main results of $[\mathrm{KLW}$. Note that in all the applications considered in this paper we will take $X$ to be an open subset of $\mathbb{R}^{d}$; however one can also work with vector spaces over other local fields and, using methods from [KT] and [G2, obtain nonArchimedean version of many results from the present paper; see $\S 6.6$ for further discussion. 
Many nontrivial examples of measures $\mu$ and maps $\mathbf{f}$ satisfying the conditions of the above theorem can be found in the paper [KLW]. For example, it is not hard to see that a measure $\mu$ on $\mathbb{R}^{n}$ is friendly (a property introduced in [KLW] ) iff it is Federer and $(\mathrm{Id}, \mu)$ is good and nonplanar (here $\mathrm{Id}$ is the identity map $\mathbb{R}^{n} \rightarrow \mathbb{R}^{n}$ ). Many measures naturally arising from geometric constructions can be shown to possess an even stronger property - such measures were referred to as 'absolutely decaying and Federer' in [KLW and as 'absolutely friendly' in $\mathrm{PV}$; many examples of those can be found in [KLW], U], SU]. It was proved in [KLW, $\S 7$ ] that if $\mu$ is absolutely decaying and Federer and $\mathbf{f}$ is nondegenerate at $\mu$-a.e. point of $\mathbb{R}^{d}$, then $(\mathbf{f}, \mu)$ is good and nonplanar. From the aforementioned facts, using Theorem 1.3 and, if necessary, compositions with affine isomorphisms, the following can be deduced:

Corollary 1.4. (a) Let $\mathcal{L}$ be a d-dimensional affine subspace of $\mathbb{R}^{n}$, let $\mu$ be a friendly measure on $\mathbb{R}^{d}$, and let $\mathbf{f}: \mathbb{R}^{d} \rightarrow \mathcal{L}$ be an affine isomorphism. Then $\omega\left(\mathbf{f}_{*} \mu\right)=\omega(\mathcal{L})$.

(b) Let $\mu$ be an absolutely decaying and Federer measure on $\mathbb{R}^{d}, \mathcal{L}$ an affine subspace of $\mathbb{R}^{n}$, and let $\mathbf{f}: \mathbb{R}^{d} \rightarrow \mathcal{L}$ be a smooth map which is nondegenerate in $\mathcal{L}$ at $\mu$-a.e. point of $\mathbb{R}^{d}$. Then $\omega\left(\mathbf{f}_{*} \mu\right)=\omega(\mathcal{L})$.

For the special case of $\mathcal{L}$ being extremal, part (a) was stated without proof in KLW, $§ 10.5]$.

\section{Weighted POSETS AND QUANTITATIVE NONDIVERGENCE}

In this section we work with mappings of weighted partially ordered sets (posets) into spaces of functions on balls in a Besicovitch metric space. Here is some relevant terminology. By a weighted poset we mean a partially ordered set $\mathfrak{P}$ together with a map $\eta: \mathfrak{P} \rightarrow \mathbb{R}_{+}$. A linearly ordered subset of $\mathfrak{P}$ will be called a flag. We will denote by $\ell(\mathfrak{P})$ the length of $\mathfrak{P}$, i.e. the cardinality of a flag with maximal cardinality. If $\mathfrak{F}$ is a subset of $\mathfrak{P}$, we let $\mathfrak{P}(\mathfrak{F})$ be the poset of elements of $\mathfrak{P} \backslash \mathfrak{F}$ comparable with every element of $\mathfrak{F}$. Note that one always has

$$
\ell(\mathfrak{P}(\mathfrak{F})) \leq \ell(\mathfrak{P})-\ell(\mathfrak{F})
$$

We will fix a metric space $X$ and consider a weighted poset $(\mathfrak{P}, \eta)$ together with a mapping $\psi$ from $\mathfrak{P}$ to the space $C(B)$ of $\mathbb{R}$-valued continuous functions on some subset $B$ of $X$, which we will denote by $s \mapsto \psi_{s}$. Given such a mapping and a positive number $\varepsilon$, we will say that a point $z \in B$ is $\varepsilon$-marked relative to $\mathfrak{P}$ if there exists a flag $\mathfrak{F}_{z} \subset \mathfrak{P}$ such that

$$
\begin{array}{ll}
\text { (M1) } \varepsilon \eta(s) \leq\left|\psi_{s}(z)\right| \leq \eta(s) & \forall s \in \mathfrak{F}_{z} ; \\
\text { (M2) }\left|\psi_{s}(z)\right| \geq \eta(s) & \forall s \in \mathfrak{P}\left(\mathfrak{F}_{z}\right) .
\end{array}
$$

We will denote the set of all such points by $\Phi(\varepsilon, \mathfrak{P})$. When it does not cause confusion, we will omit the reference to either $\mathfrak{P}, \eta$ or $\varepsilon$, and will simply say that $z$ is $\varepsilon$-marked, or marked relative to $\mathfrak{P}$.

Theorem 2.1. Let $k, N \in \mathbb{Z}_{+}$and $C, \alpha, D>0$. Suppose that we are given an $N$-Besicovitch metric space $X$, a weighted poset $(\mathfrak{P}, \eta)$, a ball $B=B(x, r)$ in $X$, a measure $\mu$ which is D-Federer on $\tilde{B} \stackrel{\text { def }}{=} B\left(x, 3^{m} r\right)$, and a mapping $\psi: \mathfrak{P} \rightarrow C(\tilde{B})$, 
$s \mapsto \psi_{s}$, such that the following holds:

(A0) $\ell(\mathfrak{P}) \leq k$;

(A1) $\forall s \in \mathfrak{P}, \quad \psi_{s}$ is $(C, \alpha)$-good on $\tilde{B}$ with respect to $\mu$;

(A2) $\forall s \in \mathfrak{P}, \quad\left\|\psi_{s}\right\|_{\mu, B} \geq \eta(s)$;

(A3) $\forall y \in \tilde{B} \cap \operatorname{supp} \mu, \quad \#\left\{s \in \mathfrak{P}|| \psi_{s}(y) \mid<\eta(s)\right\}<\infty$.

Then $\forall \varepsilon>0$ one has

$$
\mu(B \backslash \Phi(\varepsilon, \mathfrak{P})) \leq k C\left(N D^{2}\right)^{k} \varepsilon^{\alpha} \mu(B) .
$$

The proof given below is a weighted modification of the argument from [KT, $\S 5]$, which, in its turn, generalizes $\mathrm{KM}, \S 4]$. In a sense, this modification allows one to use the full strength of the construction originally introduced by Margulis Mr1, obtaining what may be considered as the optimal result (see the discussion after the proof of Theorem 2.2).

Proof. We proceed by induction on $k$. If $k=0$, the poset $\mathfrak{P}$ is empty, and for any $z \in B$ one can take $\mathfrak{F}_{z}=\varnothing$ and check that (M1) and (M2) are satisfied for all positive $\varepsilon$; thus all points of $B$ are marked. Now take $k \geq 1$ and suppose that the claim is proved for all smaller values of $k$.

Fix $C, \alpha, \rho, \mathfrak{P}, B=B(x, r)$ and $\psi$ as in the formulation of the theorem. For any $y \in B \cap \operatorname{supp} \mu$ define

$$
H(y) \stackrel{\text { def }}{=}\left\{s \in \mathfrak{P}|| \psi_{s}(y) \mid<\eta(s)\right\} ;
$$

this is a finite subset of $\mathfrak{P}$ in view of (A3). If $H(y)$ is empty, $y$ is clearly $\varepsilon$-marked for any positive $\varepsilon$ : indeed, since $\left|\psi_{s}(y)\right| \geq \eta(s)$ for all $s \in \mathfrak{P}$, one can again take $\mathfrak{F}_{y}$ to be the empty set and check that (M1) and (M2) are satisfied. Thus one only needs to consider points $y$ from the set

$$
\begin{aligned}
E & \stackrel{\text { def }}{=}\{y \in B \cap \operatorname{supp} \mu \mid H(y) \neq \varnothing\} \\
& =\left\{y \in B \cap \operatorname{supp} \mu \mid \exists s \in \mathfrak{P} \text { with }\left|\psi_{s}(y)\right|<\eta(s)\right\} .
\end{aligned}
$$

Take $y \in E$ and $s \in H(y)$, and define

$$
r_{s, y} \stackrel{\text { def }}{=} \sup \left\{t>0 \mid\left\|\psi_{s}\right\|_{\mu, B(y, t)}<\eta(s)\right\} .
$$

It follows from the continuity of functions $\psi_{s}$ that for small enough positive $t$ one has $\left\|\psi_{s}\right\|_{\mu, B(y, t)}<\eta(s)$, hence $r_{s, y}>0$. Denote $B\left(y, r_{s, y}\right)$ by $B_{s, y}$. From (A2) it is clear that $B_{s, y}$ does not contain $B$; therefore one has $r_{s, y}<2 r$. Note also that (2.2) immediately implies that

$$
\left\|\psi_{s}\right\|_{\mu, B_{s, y}} \leq \eta(s) .
$$

Now for any $y \in E$ choose an element $s_{y}$ of $H(y)$ such that $r_{s_{y}, y} \geq r_{s, y}$ for all $s \in H(y)$ (this can be done since $H(y)$ is finite). For brevity let us denote $r_{s_{y}, y}$ by $r_{y}$ and $B_{s_{y}, y}=\bigcup_{s \in H(y)} B_{s, y}$ by $B_{y}$. Also let us denote the poset $\mathfrak{P}\left(\left\{s_{y}\right\}\right)$ by $\mathfrak{P}_{y}$.

The next claim allows one to show a point $z \in B_{y}$ to be marked relative to $\mathfrak{P}$ once it is marked relative to $\mathfrak{P}_{y}$. Namely, fix $\varepsilon>0$ and $y \in E$, and take $z$ such that

$$
z \in B_{y} \cap \operatorname{supp} \mu \cap \Phi\left(\varepsilon, \mathfrak{P}_{y}\right) \text { and }\left|\psi_{s_{y}}(z)\right| \geq \varepsilon \eta\left(s_{y}\right) .
$$

Then we claim that $z$ belongs to $\Phi(\varepsilon, \mathfrak{P})$; equivalently,

$$
\left(B_{y} \cap \operatorname{supp} \mu\right) \backslash \Phi(\varepsilon, \mathfrak{P}) \subset\left(B_{y} \backslash \Phi\left(\varepsilon, \mathfrak{P}_{y}\right)\right) \cup\left\{x \in B_{y}|| \psi_{s_{y}}(x) \mid<\varepsilon \eta\left(s_{y}\right)\right\} .
$$


Indeed, take $z$ as in $(2.4)$; by definition of $\Phi\left(\varepsilon, \mathfrak{P}_{y}\right)$, there exists a flag $\mathfrak{F}_{y, z} \subset \mathfrak{P}_{y}$ such that

$$
\varepsilon \eta(s) \leq\left|\psi_{s}(z)\right| \leq \eta(s) \quad \forall s \in \mathfrak{F}_{y, z}
$$

and

$$
\left|\psi_{s}(z)\right| \geq \eta(s) \quad \forall s \in \mathfrak{P}_{y}\left(\mathfrak{F}_{y, z}\right) .
$$

Put $\mathfrak{F}_{z} \stackrel{\text { def }}{=} \mathfrak{F}_{y, z} \cup\left\{s_{y}\right\}$. Then $\mathfrak{P}\left(\mathfrak{F}_{z}\right)=\mathfrak{P}_{y}\left(\mathfrak{F}_{y, z}\right)$, so (M2) immediately follows from (2.7). Property (M1) for $s \neq s_{y}$ is given by (2.6), and for $s=s_{y}$ by (2.4) and (2.3). Thus (2.5) is proved.

Note that for any $y \in E$ one clearly has $r_{y}<2 r$, which in particular implies that $B_{y} \subset B(x, 3 r)$. We are going to fix some $r_{y}^{\prime}$ strictly between $r_{y}$ and $\min \left(2 r, 3 r_{y}\right)$, and denote $B\left(y, r_{y}^{\prime}\right)$ by $B_{y}^{\prime}$. Clearly one has

$$
\left\|\psi_{s}\right\|_{\mu, B_{y}^{\prime}} \geq \eta(s) \text { for any } y \in E \text { and } s \in \mathfrak{P} .
$$

(Indeed, the definition of $r_{y}$ and (2.2) imply the above inequality for any $s \in H(y)$, and it obviously holds if $s \notin H(y)$.)

Now observe that $\mathfrak{P}_{y}, B_{y}^{\prime}$ and $\tilde{B}_{y}^{\prime} \stackrel{\text { def }}{=} B\left(y, 3^{k-1} r_{y}^{\prime}\right)$ satisfy properties

- (A0) with $k$ replaced by $k-1 \quad-\quad$ in view of (2.1);

- (A2)

- (A1) and (A3)

- $\quad$ in view of $(2.8)$;

- since

$$
\tilde{B}_{y}^{\prime}=B\left(y, 3^{k-1} r_{y}^{\prime}\right) \subset B\left(x, 3^{k-1} r_{y}^{\prime}+r\right) \subset B\left(x,\left(2 \cdot 3^{k-1}+1\right) r\right) \subset B\left(x, 3^{k} r\right)=\tilde{B} .
$$

Therefore one has

$$
\begin{aligned}
\mu\left(B_{y} \backslash \Phi\left(\varepsilon, \mathfrak{P}_{y}\right)\right) & \leq \mu\left(B_{y}^{\prime} \backslash \Phi\left(\varepsilon, \mathfrak{P}_{y}\right)\right) \leq(k-1) C\left(N D^{2}\right)^{k-1} \varepsilon^{\alpha} \mu\left(B_{y}^{\prime}\right) \\
& \leq D(k-1) C\left(N D^{2}\right)^{k-1} \varepsilon^{\alpha} \mu\left(B_{y}\right)
\end{aligned}
$$

by the induction assumption and the Federer property of $\mu$. On the other hand, in view of $\psi_{s_{y}}$ being $(C, \alpha)$-good on $\tilde{B} \supset B_{y}^{\prime}$ with respect to $\mu$, one can write

$$
\begin{array}{r}
\mu\left(\left\{x \in B_{y}|| \psi_{s_{y}}(x) \mid<\varepsilon \eta\left(s_{y}\right)\right\}\right) \leq \mu\left(\left\{x \in B_{y}^{\prime}|| \psi_{s_{y}}(x) \mid<\varepsilon \eta\left(s_{y}\right)\right\}\right) \\
\leq C\left(\frac{\varepsilon \eta\left(s_{y}\right)}{\left\|\psi_{s_{y}}\right\|_{\mu, B_{y}^{\prime}}}\right)^{\alpha} \mu\left(B_{y}^{\prime}\right) \underset{(2.8)}{\leq} C \varepsilon^{\alpha} \mu\left(B_{y}^{\prime}\right) \underset{\text { Federer }}{\leq} C D \varepsilon^{\alpha} \mu\left(B_{y}\right) .
\end{array}
$$

Now recall that we need to estimate the measure of $E \backslash \Phi(\varepsilon, \mathfrak{P})$. For any $y \in E$, in view of (2.5), (2.9) and (2.10) one has

$$
\begin{aligned}
\mu\left(B_{y} \backslash \Phi(\varepsilon, \mathfrak{P})\right) & \leq C\left((k-1) N^{k-1} D^{2 k-1}+D\right) \varepsilon^{\alpha} \mu\left(B_{y}\right) \\
& \leq k C N^{k-1} D^{2 k-1} \varepsilon^{\alpha} \mu\left(B_{y}\right) .
\end{aligned}
$$

Finally, consider the covering $\left\{B_{y} \mid y \in E\right\}$ of $E$, choose a countable subset $Y$ of $E$ such that the multiplicity of the subcovering $\left\{B_{y} \mid y \in Y\right\}$ is at most $N$, and write

$$
\sum_{y \in Y} \mu\left(B_{y}\right) \leq N \mu\left(\bigcup_{y \in Y} B_{y}\right) \leq N \mu(B(x, 3 r)) \leq N D \mu(B)
$$


Therefore the measure of $E \backslash \Phi(\varepsilon, \mathfrak{P})$ is bounded from above by

$$
\begin{gathered}
\sum_{y \in Y} \mu\left(B_{y} \backslash \Phi(\varepsilon, \mathfrak{P})\right) \underset{(2.11)}{\leq} k C N^{k-1} D^{2 k-1} \varepsilon^{\alpha} \sum_{y \in Y} \mu\left(B_{y}\right) \\
\leq k C\left(N D^{2}\right)^{k} \varepsilon^{\alpha} \mu(B) \\
(2.12)
\end{gathered}
$$

and the theorem is proven.

We now apply Theorem 2.1 to the (appropriately weighted) poset

$$
\mathfrak{P}_{k} \stackrel{\text { def }}{=}\left\{\text { nonzero primitive subgroups of } \mathbb{Z}^{k}\right\} \text {, }
$$

with the inclusion relation (recall that a discrete subgroup $\Gamma \subset \mathbb{Z}^{k}$ is called primitive if $\Gamma=\Gamma_{\mathbb{R}} \cap \mathbb{Z}^{k}$ ), and prove a general version of Theorem 0.2 .

Theorem 2.2. Let $k, N \in \mathbb{N}$ and $C, D, \alpha, \rho>0$, and suppose we are given an $N$-Besicovitch metric space $X$, a ball $B=B\left(x_{0}, r_{0}\right) \subset X$, a measure $\mu$ which is $D$-Federer on $\tilde{B} \stackrel{\text { def }}{=} B\left(x_{0}, 3^{k} r_{0}\right)$, and a map $h: \tilde{B} \rightarrow \mathrm{GL}_{k}(\mathbb{R})$. Assume that the following two conditions hold:

[2.2-i] $\forall \Gamma \in \mathfrak{P}_{k}$, the function $x \mapsto\|h(x) \Gamma\|$ is $(C, \alpha)$-good on $\tilde{B}$ w.r.t. $\mu$;

$[2.2-\mathrm{ii}] \forall \Gamma \in \mathfrak{P}_{k}, \quad\|h(\cdot) \Gamma\|_{\mu, B} \geq \rho^{\mathrm{rk}(\Gamma)}$.

Then for any positive $\varepsilon \leq \rho$ one has

$$
\mu\left(\left\{x \in B \mid h(x) \mathbb{Z}^{k} \notin K_{\varepsilon}\right\}\right) \leq k C\left(N D^{2}\right)^{k}\left(\frac{\varepsilon}{\rho}\right)^{\alpha} \mu(B) .
$$

Proof. We let $\mathfrak{P}=\mathfrak{P}_{k}$ and for all $\Gamma \in \mathfrak{P}$ define $\psi_{\Gamma}(\cdot) \stackrel{\text { def }}{=}\|h(\cdot) \Gamma\|$ and $\eta(\Gamma)=\rho^{\operatorname{rk}(\Gamma)}$. It is easy to verify that $(\mathfrak{P}, \eta)$ and $\psi$ satisfy properties (A0)-(A3) of Theorem 2.1 . Indeed, (A0) holds since any two primitive subgroups are either incomparable or have the same rank, (A1) is given by [2.2-i] and (A2) by [2.2-ii]. To check (A3) it suffices to observe that for any $x$, since $\Lambda\left(h(x) \mathbb{Z}^{k}\right)$ is discrete in $\mathbb{R}^{k}$, the number of primitive subgroups $\Gamma$ of $\mathbb{Z}^{k}$ for which $\|h(x) \Gamma\| \leq 1$ is finite.

In view of Theorem 2.1, it remains to prove that a point $x \in B$ with $h(x) \mathbb{Z}^{k} \notin K_{\varepsilon}$ cannot be $\frac{\varepsilon}{\rho}$-marked; in other words,

$$
\Phi(\varepsilon / \rho, \mathfrak{P}) \subset\left\{x \in B \mid\|\mathbf{v}\| \geq \varepsilon \text { for all } \mathbf{v} \in \mathbb{Z}^{k} \backslash\{0\}\right\} .
$$

Take an $\frac{\varepsilon}{\rho}$-marked point $x \in B$, and let $\{0\}=\Gamma_{0} \subsetneq \Gamma_{1} \subsetneq \cdots \subsetneq \Gamma_{j}=\mathbb{Z}^{k}$ be all the elements of $\mathfrak{F}_{x} \cup\left\{\{0\}, \mathbb{Z}^{k}\right\}$. Properties (M1) and (M2) translate into:

$$
\frac{\varepsilon}{\rho} \cdot \rho^{\mathrm{rk}\left(\Gamma_{i}\right)} \leq\left\|h(x) \Gamma_{i}\right\| \leq \rho^{\mathrm{rk}\left(\Gamma_{i}\right)} \quad \forall i=0, \ldots, j-1,
$$

and

$$
\|h(x) \Gamma\| \geq \rho^{\mathrm{rk}(\Gamma)} \quad \forall \Gamma \in \mathfrak{P}\left(\mathfrak{S}_{x}\right) .
$$

(Even though $\Gamma_{0}=\{0\} \notin \mathfrak{P}$, it is clear that it also satisfies (2.14).)

Take any $\mathbf{v} \in \mathbb{Z}^{k} \backslash\{0\}$. Then there exists $i, 1 \leq i \leq j$, such that $\mathbf{v} \in \Gamma_{i} \backslash \Gamma_{i-1}$. Denote $\left(\Gamma_{i-1}+\mathbb{Z} \mathbf{v}\right)_{\mathbb{R}} \cap \mathbb{Z}^{k}$ by $\Gamma$. Clearly it is a primitive subgroup of $\mathbb{Z}^{k}$ satisfying $\Gamma_{i-1} \subset \Gamma \subset \Gamma_{i}$, therefore $\Gamma \in \mathfrak{F}_{x} \cup \mathfrak{P}\left(\mathfrak{F}_{x}\right)$. Now one can use properties (2.14) and (2.15) to deduce that

$$
\|h(x) \Gamma\| \geq \min \left(\frac{\varepsilon}{\rho} \cdot \rho^{\mathrm{rk}(\Gamma)}, \rho^{\mathrm{rk}(\Gamma)}\right)=\varepsilon \rho^{\mathrm{rk}(\Gamma)-1}=\varepsilon \rho^{\mathrm{rk}\left(\Gamma_{i-1}\right)} .
$$


On the other hand, from the submultiplicativity of the covolume it follows that $\|h(x) \Gamma\| \leq\left\|h(x) \Gamma_{i-1}\right\| \cdot\|h(x) \mathbf{v}\|$. Thus

$$
\|h(x) \mathbf{v}\| \geq \frac{\|h(x) \Gamma\|}{\left\|h(x) \Gamma_{i-1}\right\|} \text { by (2.14) and (2.16) } \frac{\varepsilon \rho^{\mathrm{rk}\left(\Gamma_{i-1}\right)}}{\rho^{\mathrm{rk}\left(\Gamma_{i-1}\right)}}=\varepsilon .
$$

This shows (2.13) and completes the proof of the theorem.

In order to better understand the difference between this theorem and its predecessors (proved in $[\mathrm{KM}$, , $\mathrm{KLW},[\mathrm{KT}]$ ), let us draw a corollary from it. Namely, suppose that $X, \mu$ and $B$ are as in the above theorem, that $h$ satisfies [2.2-i] with some $C, \alpha$ (for example, $X=\mathbb{R}^{d}, \mu=\lambda$ and $h$ is a polynomial map), and that for some small positive $\varepsilon$, the relative measure of $x \in B$ for which $h(x) \mathbb{Z}^{k} \notin K_{\varepsilon}$ is at least $1 / 2$. Then Theorem 2.2 asserts that there exists a subgroup $\Gamma$ of $\mathbb{Z}^{k}$ such that $\|h(\cdot) \Gamma\|_{\mu, B}<\rho^{\mathrm{rk}(\Gamma)}$, where $\rho=\left(2 k C\left(N D^{2}\right)^{k}\right)^{1 / \alpha} \varepsilon$. Consequently, in view of Minkowski's Lemma, the whole 'trajectory' $h(B \cap \operatorname{supp} \mu) \mathbb{Z}^{k}$ must be contained in the complement to $K_{\text {const } \varepsilon \varepsilon}$, with the constant depending only on $D, N, C, \alpha$ and $k$. In other words, it must stay at a (uniformly) bounded distance from the complement to $K_{\varepsilon}$. Note that using previously known results it was only possible to conclude that $h(B \cap \operatorname{supp} \mu) \mathbb{Z}^{k}$ must be outside of $K_{\text {const } \cdot \varepsilon^{1 /(k-1)}}$, a compact set of diameter approximately $(k-1)$ times smaller than that of $K_{\varepsilon}$.

When it comes to number-theoretic applications, the crucial advantage is that replacing $\rho$ by $\rho^{\mathrm{rk}(\Gamma)}$ makes [2.2-ii] easier to check. This will be demonstrated in the next section, where Theorem 2.2 will be applied to $h$ as in (0.4).

\section{An application to metric Diophantine approximation}

We recall some notation and terminology introduced in the beginning of this paper, as well as in the paper [K2]. For $m, n \in \mathbb{N}$ and $v>0$, we denote by $\mathcal{W}_{v}$ the set of $A \in M_{m, n}$ for which there are infinitely many $\mathbf{q} \in \mathbb{Z}^{n}$ such that (0.5) holds for some $\mathbf{p} \in \mathbb{Z}^{m}$. The dimensionality of the matrices will be clear from the context. The Diophantine exponent $\omega(A)$ of $A$ defined in the Introduction is equal to

$$
\omega(A)=\sup \left\{v \mid A \in \mathcal{W}_{v}\right\} .
$$

Clearly $\mathcal{W}_{u} \subset \mathcal{W}_{v}$ if $u \geq v$. We will also use the notation

$$
\mathcal{W}_{v}^{+} \stackrel{\text { def }}{=} \bigcup_{u>v} \mathcal{W}_{u}=\{A \mid \omega(A)>v\}
$$

Note that the definition of $\omega(A)$, unlike that of the sets $\mathcal{W}_{v}$, does not depend on the choice of norms on $\mathbb{R}^{m}$ and $\mathbb{R}^{n}$.

Although there are many interesting and unsolved Diophantine problems related to the space of $m \times n$ matrices, we specialize to the case $m=1$, that is, consider Diophantine properties of vectors (= row matrices) $\mathbf{y} \in \mathbb{R}^{n}$. With some abuse of notation, we will view integer vectors $\mathbf{q} \in \mathbb{Z}^{n}$ as column vectors, so that yq stands for $y_{1} q_{1}+\cdots+y_{n} q_{n}$.

Now let us describe a correspondence, dating back to Sc1] and Da3, between approximation properties of vectors $\mathbf{y} \in \mathbb{R}^{n}$ and dynamics of certain trajectories in $\Omega_{n+1}$. Given a row vector $\mathbf{y} \in \mathbb{R}^{n}$ one defines

$$
u_{\mathbf{y}} \stackrel{\text { def }}{=}\left(\begin{array}{cc}
1 & \mathbf{y} \\
0 & I_{n}
\end{array}\right)
$$


and considers the lattice $u_{\mathbf{y}} \mathbb{Z}^{n+1}$ in $\mathbb{R}^{n+1}$, that is, the collection of vectors of the form $\left(\begin{array}{c}\mathbf{y q}+p \\ \mathbf{q}\end{array}\right)$, where $p \in \mathbb{Z}$ and $\mathbf{q} \in \mathbb{Z}^{n}$. Then one can read Diophantine properties of $\mathbf{y}$ from the behavior of the trajectory $F u_{\mathbf{y}} \mathbb{Z}^{n+1}$ in the space of lattices, where

$$
F=\left\{g_{t} \mid t \geq 0\right\}, \quad \text { with } g_{t}=\operatorname{diag}\left(e^{t}, e^{-t / n}, \ldots, e^{-t / n}\right),
$$

is a one-parameter subsemigroup of $\mathrm{SL}_{n+1}(\mathbb{R})$ which expands the first coordinate and uniformly contracts the last $n$ coordinates of vectors in $\mathbb{R}^{n+1}$.

The following elementary lemma was proved in [K2].

Lemma 3.1. Suppose we are given a set $E \subset \mathbb{R}^{2}$ which is discrete and homogeneous with respect to positive integers, that is, $k E \subset E$ for any $k \in \mathbb{N}$. Also take $a, b>0$, $v>a / b$, and define $c$ by

$$
c=\frac{b v-a}{v+1} \quad \Leftrightarrow \quad v=\frac{a+c}{b-c} .
$$

Then the following are equivalent:

[3.1-i] there exist $(x, z) \in E$ with arbitrarily large $|z|$ such that $|x| \leq|z|^{-v}$;

[3.1-ii] there exist arbitrarily large $t>0$ such that for some $(x, z) \in E \backslash\{0\}$ one has

$$
\max \left(e^{a t}|x|, e^{-b t}|z|\right) \leq e^{-c t} .
$$

Taking $v>n, \mathbf{y} \in \mathbb{R}^{n}$ and

$$
E=\left\{(\mathbf{y q}+p,\|\mathbf{q}\|) \mid(p, \mathbf{q}) \in \mathbb{Z}^{n+1}\right\},
$$

one notices that [3.1-i] is equivalent to $\mathbf{y} \in \mathcal{W}_{v}$. On the other hand, choosing $a=1$ and $b=1 / n$ one sees that [3.1-ii] amounts to

$$
g_{t} u_{\mathbf{y}} \mathbb{Z}^{n+1} \notin K_{e^{-c t}} \text { for an unbounded set of } t \in \mathbb{R}_{+},
$$

where

$$
c=\frac{v-n}{n(v+1)} \quad \Leftrightarrow \quad v=\frac{n(1+c)}{1-n c} .
$$

Thus, if we define $\gamma(\mathbf{y})$ to be the supremum of all $c$ for which (3.3) holds, or, equivalently,

$$
\gamma(\mathbf{y})=\sup \left\{c \mid g_{t} u_{\mathbf{y}} \mathbb{Z}^{n+1} \notin K_{e^{-c t}} \text { for infinitely many } t \in \mathbb{N}\right\}
$$

(in $\mathrm{K} 2$ this quantity was called the growth exponent of $u_{\mathbf{y}} \mathbb{Z}^{n+1}$ with respect to $F)$, then we have the equality

$$
\omega(\mathbf{y})=n \frac{1+\gamma(\mathbf{y})}{1-n \gamma(\mathbf{y})}
$$

(cf. [K2, Corollary 2.3]).

Now let us turn to computing Diophantine exponents of measures. If $\nu$ is a measure on $\mathbb{R}^{n}$ and $v \geq n$, one has $\omega(\nu) \leq v$ iff $\nu\left(\mathcal{W}_{u}\right)=0$ for any $u>v$. In view of the above discussion, this amounts to saying that for any $d>c$ where $c$ is given by $(3.4)$,

$$
\nu\left(\left\{\mathbf{y} \mid g_{t} u_{\mathbf{y}} \mathbb{Z}^{n+1} \notin K_{e^{-d t}} \text { for an unbounded set of } t \in \mathbb{R}_{+}\right\}\right)=0 .
$$


This is easily seen to be equivalent to

$$
\forall d>c, \quad \nu\left(\left\{\mathbf{y} \mid g_{t} u_{\mathbf{y}} \mathbb{Z}^{n+1} \notin K_{e^{-d t}} \text { for infinitely many } t \in \mathbb{N}\right\}\right)=0,
$$

and, in view of the Borel-Cantelli Lemma, a condition sufficient for the latter is

$$
\sum_{t=1}^{\infty} \nu\left(\left\{\mathbf{y} \mid g_{t} u_{\mathbf{y}} \mathbb{Z}^{n+1} \notin K_{e^{-d t}}\right\}\right)<\infty \quad \forall d>c .
$$

This is precisely where the measure estimates discussed in the previous section come in.

Proposition 3.2. Let $X$ be a Besicovitch metric space, $B=B(x, r) \subset X$ a ball, $\mu$ a measure which is $D$-Federer on $\tilde{B} \stackrel{\text { def }}{=} B\left(x, 3^{n+1} r\right)$ for some $D>0$, and $\mathbf{f}$ a continuous map from $\tilde{B}$ to $\mathbb{R}^{n}$. Also take $c \geq 0$ and assume that:

[3.2-i] $\exists C, \alpha>0$ such that all the functions $x \mapsto\left\|g_{t} u_{\mathbf{f}(x)} \Gamma\right\|, \Gamma \in \mathfrak{P}_{n+1}$, are $(C, \alpha)$-good on $\tilde{B}$ w.r.t. $\mu$;

[3.2-ii] for any $d>c$ there exists $T=T(d)>0$ such that for any $t \geq T$ and any $\Gamma \in \mathfrak{P}_{n+1}$ one has

$$
\left\|g_{t} u_{\mathbf{f}(\cdot)} \Gamma\right\|_{\mu, B} \geq e^{-\mathrm{rk}(\Gamma) d t} .
$$

Then $\omega\left(\mathbf{f}_{*}\left(\left.\mu\right|_{B}\right)\right) \leq v$, where $v$ is related to $c$ via (3.4).

Proof. As was observed in the course of the preceding discussion, it suffices to show (3.6) for $\nu=\mathbf{f}_{*}\left(\left.\mu\right|_{B}\right)$. We now proceed to verify that the map $h=g_{t} u_{\mathbf{f}}$ satisfies the assumptions of Theorem 2.2, with $k=n+1$. Condition [2.2-i] clearly coincides with [3.2-i]. For the other condition, we take $d>c$ and $\rho=e^{-\frac{c+d}{2} t}$, so that [3.2-ii] implies [2.2-ii] for any $t>T\left(\frac{c+d}{2}\right)$. Therefore, by Theorem 2.2,

$$
\begin{aligned}
\nu\left(\left\{\mathbf{y} \mid g_{t} u_{\mathbf{y}} \mathbb{Z}^{n+1} \notin K_{e^{-d t}}\right\}\right) & =\mu\left(\left\{x \in B \mid h(x) \mathbb{Z}^{n+1} \notin K_{e^{-d t}}\right\}\right) \\
& \leq(n+1) C\left(N D^{2}\right)^{n+1}\left(e^{-d t} e^{\frac{c+d}{2} t}\right)^{\alpha} \mu(B) \\
& =\text { const } \cdot e^{-\alpha \frac{d-c}{2} t}
\end{aligned}
$$

for all but finitely many $t \in \mathbb{N}$. This readily implies (3.6).

Remark 3.3. Note that both [3.2-i] and [3.2-ii] trivially hold for $\Gamma$ of rank 0 and $n+1$, and also that the validity of those conditions for all primitive $\Gamma$ is equivalent to their validity for all subgroups. It will be convenient to denote by $\mathcal{S}_{k, j}$ the set of all subgroups of $\mathbb{Z}^{k}$ of rank $j$. Thus, to apply Proposition 3.2 it will be enough to check $\left[3.2\right.$-i,ii] for all $\Gamma \in \mathcal{S}_{n+1, j}, j=1, \ldots, n$.

\section{Computing Diophantine exponents of measures}

In this section we use Proposition 3.2 to prove Theorem 1.3, that is, for a given $v \geq n$, write necessary and sufficient conditions for Diophantine exponents of certain measures to be not greater than $v$. For this we need to understand to what extent the two conditions in the above proposition are necessary. While not much can be said about the first one, it turns out that assumption [3.2-ii] is in fact necessary for the conclusion of Proposition 3.2. Furthermore, the consequences of [3.2-ii] not being true are surprisingly strong. 
Lemma 4.1. Let $\mu$ be a measure on a set $B$, take $c, v>0$ related via (3.4), and let $\mathbf{f}$ be a map from $B$ to $\mathbb{R}^{n}$ such that $\left[3.2\right.$-ii] does not hold. Then $\mathbf{f}(B \cap \operatorname{supp} \mu) \subset \mathcal{W}_{u}$ for some $u>v$.

We remark that the negation of the conclusion of Proposition 3.2 is much weaker: it simply amounts to saying that for some $u>v$ the set $\left\{x \in B \mid \mathbf{f}(x) \in \mathcal{W}_{u}\right\}$ has positive measure.

Proof. The assumption of the lemma says that there exists $d>c$ such that one has

$$
\sup _{x \in B \cap \operatorname{supp} \mu}\left\|g_{t} u_{\mathbf{f}(x)} \Gamma\right\|<e^{-\operatorname{rk}(\Gamma) d t}
$$

for arbitrarily large $t$ (and $\Gamma \subset \mathbb{Z}^{n+1}$ dependent on $t$ ). In other words, there exists $1 \leq j \leq n$, a sequence $t_{i} \rightarrow \infty$ and a sequence of subgroups $\Gamma_{i} \in \mathcal{S}_{n+1, j}$ such that for any $x \in B \cap \operatorname{supp} \mu$ one has $\left\|g_{t_{i}} u_{\mathbf{f}(x)} \Gamma_{i}\right\|<e^{-j d t_{i}}$. But in view of Minkowski's Lemma, this means that for any $i$ and any $x \in B \cap \operatorname{supp} \mu$ there is a nonzero vector $\mathbf{v} \in g_{t_{i}} u_{\mathbf{f}(x)} \Gamma_{i}$ with $\|\mathbf{v}\|<2^{j} e^{-d t_{i}}$. Hence

$$
g_{t_{i}} u_{\mathbf{f}(x)} \mathbb{Z}^{n+1} \notin K_{2^{j} e^{-d t_{i}}},
$$

which implies that $\gamma(\mathbf{f}(x)) \geq d$, finishing the proof in view of (3.5).

It is worthwhile to point out that it is precisely the above argument that requires a strengthening of the quantitative nondivergence obtained in $\S 2$. Previously available techniques could only yield $\gamma(\mathbf{f}(x)) \geq d / n$, which was enough in the case $v=n$ $\Leftrightarrow c=0$, but not in the general case.

To write [3.2-ii] in a more convenient form, instead of discrete subgroups $\Gamma$ of $\mathbb{R}^{k}$ we will work with elements $\mathbf{w} \in \bigwedge\left(\mathbb{R}^{k}\right)$ representing them, saying that $\mathbf{w}$ represents $\Gamma \neq\{0\}$ if $\mathbf{w}=\mathbf{v}_{1} \wedge \cdots \wedge \mathbf{v}_{j}$ where $\mathbf{v}_{1}, \ldots, \mathbf{v}_{j}$ is a basis of $\Gamma$ as a $\mathbb{Z}$-module. Clearly $\mathbf{w}$ representing $\Gamma$ is defined up to a sign, hence $\|\Gamma\|=\|\mathbf{w}\|$. Now let us reproduce a computation (first done in $[\mathrm{KM}]$ ) of coordinates of $g_{t} u_{\mathbf{f}(x)} \Gamma$ with respect to the standard basis of $\bigwedge\left(\mathbb{R}^{n+1}\right)$. For the rest of this section let us denote $\mathbb{R}^{n+1}$ by $V$, its standard basis by $\mathbf{e}_{0}, \mathbf{e}_{1}, \ldots, \mathbf{e}_{n}$, and the space spanned by $\mathbf{e}_{1}, \ldots, \mathbf{e}_{n}$ by $V_{0}$. For $I=\left\{i_{1}, \ldots, i_{j}\right\} \subset\{0, \ldots, n\}, i_{1}<i_{2}<\cdots<i_{j}$, let

$$
\mathbf{e}_{I} \stackrel{\text { def }}{=} \mathbf{e}_{i_{1}} \wedge \cdots \wedge \mathbf{e}_{i_{j}} \in \wedge^{j}(V),
$$

with the convention $\mathbf{e}_{\varnothing}=1$; then $\left\{\mathbf{e}_{I} \mid \# I=j\right\}$ is a basis of $\bigwedge^{j}(V)$, and we extend the Euclidean structure $\langle\cdot, \cdot\rangle$ from $V$ to its exterior powers so that this basis becomes orthonormal.

Since the action of $u_{\mathbf{y}}$ leaves $\mathbf{e}_{0}$ invariant and sends $\mathbf{e}_{i}, i>0$, to $\mathbf{e}_{i}+y_{i} \mathbf{e}_{0}$, one can write 1

$$
u_{\mathbf{y}} \mathbf{e}_{I}=\left\{\begin{array}{lc}
\mathbf{e}_{I} & \text { if } 0 \in I, \\
\mathbf{e}_{I}+\sum_{i \in I}\left\langle\mathbf{e}_{i} \wedge \mathbf{e}_{I \backslash\{i\}}, \mathbf{e}_{I}\right\rangle y_{i} \mathbf{e}_{I \cup\{0\} \backslash\{i\}} & \text { otherwise. }
\end{array}\right.
$$

Therefore $\mathbf{w} \in \bigwedge^{j}(V)$ is sent to

$$
u_{\mathbf{y}} \mathbf{w}=\sum_{I \subset\{1, \ldots, n\}}\left\langle\mathbf{e}_{I}, \mathbf{w}\right\rangle \mathbf{e}_{I}+\sum_{J \subset\{1, \ldots, n\}}\left(\sum_{i=0}^{n}\left\langle\mathbf{e}_{i} \wedge \mathbf{e}_{J}, \mathbf{w}\right\rangle y_{i}\right) \mathbf{e}_{\{0\} \cup J}
$$

\footnotetext{
${ }^{1}$ The quantity $\left\langle\mathbf{e}_{i} \wedge \mathbf{e}_{I \backslash\{i\}}, \mathbf{e}_{I}\right\rangle$ in (4.1), which is equal to 1 (resp., -1 ) if the number of elements of $I$ strictly between 0 and $i$ is even (resp., odd), was denoted by $(-1)^{\ell(I, i)}$ in $\mathrm{K} 2$.
} 
(where it is understood that $\# I=j, \# J=j-1$, and $y_{0}=1$ ). Note that the first sum in (4.2) is the image of $\mathbf{w}$ under the orthogonal projection from $\wedge^{j}(V)$ onto $\bigwedge^{j}\left(V_{0}\right)$, which (the projection) we will denote by $\pi$. On the other hand, each term in the second sum is orthogonal to $\bigwedge\left(V_{0}\right)$. To simplify (4.2), let us define a linear map $\mathbf{c}$ from $\bigwedge^{j}(V)$ to $\left(\bigwedge^{j-1}\left(V_{0}\right)\right)^{n+1}$ by setting the $i$ th component of $\mathbf{c}(\mathbf{w})$, $i=0,1, \ldots, n$, equal to

$$
\mathbf{c}(\mathbf{w})_{i} \stackrel{\text { def }}{=} \sum_{\substack{J \subset\{1, \ldots, n\} \\ \# J=j-1}}\left\langle\mathbf{e}_{i} \wedge \mathbf{e}_{J}, \mathbf{w}\right\rangle \mathbf{e}_{J} .
$$

For example, the choice $i=0$ gives $\mathbf{c}(\mathbf{w})_{0}=\sum_{I \ni 0}\left\langle\mathbf{e}_{I}, \mathbf{w}\right\rangle \mathbf{e}_{I \backslash\{0\}}$, so that

$$
\mathbf{e}_{0} \wedge \mathbf{c}(\mathbf{w})_{0}=\mathbf{w}-\pi(\mathbf{w}) \text {. }
$$

In particular, the kernel of $\mathbf{w} \mapsto \mathbf{c}(\mathbf{w})_{0}$ is $\bigwedge^{j}\left(V_{0}\right)$, that is, the orthogonal complement to $\mathbf{e}_{0} \wedge \bigwedge^{j-1}\left(V_{0}\right)$. Likewise, for every $i$ the kernel of $\mathbf{w} \mapsto \mathbf{c}(\mathbf{w})_{i}$ is the orthogonal complement to $\mathbf{e}_{i} \wedge \bigwedge^{j-1}\left(V_{0}\right)$, which implies that the kernel of $\mathbf{c}$ is trivial.

With this notation, (4.2) can be rewritten as

$$
u_{\mathbf{y}} \mathbf{w}=\pi(\mathbf{w})+\mathbf{e}_{0} \wedge \tilde{\mathbf{y}} \mathbf{c}(\mathbf{w}),
$$

where $\tilde{\mathbf{y}}$ stands for the row vector $\left(1, y_{1}, \ldots, y_{n}\right)$, and the product in $\tilde{\mathbf{y}} \mathbf{c}(\mathbf{w})$ is the formal matrix multiplication (of the row vector $\tilde{\mathbf{y}} \in \mathbb{R}^{n+1}$ and the column vector $\left.\mathbf{c}(\mathbf{w}) \in(\bigwedge(V))^{n+1}\right)$. In other words,

$$
u_{\mathbf{y}} \mathbf{w}=\pi(\mathbf{w})+\mathbf{e}_{0} \wedge \sum_{i=0}^{n} y_{i} \mathbf{c}(\mathbf{w})_{i} \underset{(4.4)}{=} \mathbf{w}+\mathbf{e}_{0} \wedge \sum_{i=1}^{n} y_{i} \mathbf{c}(\mathbf{w})_{i} .
$$

Since $\bigwedge^{j}\left(V_{0}\right)$ and its orthogonal complement are eigenspaces of $g_{t}$ with eigenvalues $e^{-\frac{j}{n} t}$ and $e^{\frac{n+1-j}{n} t}$, respectively, one can write

$$
g_{t} u_{\mathbf{y}} \mathbf{w}=e^{-\frac{j}{n} t} \pi(\mathbf{w})+e^{\frac{n+1-j}{n} t} \mathbf{e}_{0} \wedge \tilde{\mathbf{y}} \mathbf{c}(\mathbf{w}) ;
$$

thus, up to a uniform constant,

$$
\left\|g_{t} u_{\mathbf{f}(\cdot)} \Gamma\right\|_{\mu, B}=\max \left(e^{\frac{n+1-j}{n} t}\|\tilde{\mathbf{f}}(\cdot) \mathbf{c}(\mathbf{w})\|_{\mu, B}, e^{-\frac{j}{n} t}\|\pi(\mathbf{w})\|\right),
$$

where $\Gamma \in \mathcal{S}_{n+1, j}$ is represented by $\mathbf{w}$, and we use the notation $\tilde{\mathbf{f}} \stackrel{\text { def }}{=}\left(1, f_{1}, \ldots, f_{n}\right)$.

Observe that from the above one can already extract a nice lower bound for (4.6) whenever the restrictions of $1, f_{1}, \ldots, f_{n}$ to $B \cap \operatorname{supp} \mu$ are linearly independent. Indeed, then the correspondence $\mathbf{v} \mapsto\|\tilde{\mathbf{f}}(\cdot) \mathbf{v}\|_{\mu, B}$ yields a norm on $(\Lambda(V))^{n+1}$, which is obviously equivalent to the standard (Euclidean) norm. Thus, up to a (uniform in $\mathbf{w}$ ) constant, the expression (4.6) is bounded from below by $\|\mathbf{c}(\mathbf{w})\|$. Since $\mathbf{c}$ has trivial kernel and maps $\bigwedge\left(V_{\mathbb{Z}}\right)$ into $\left(\bigwedge\left(V_{\mathbb{Z}}\right)\right)^{n+1}$, one has $\|\mathbf{c}(\mathbf{w})\| \geq 1$ for any nonzero $\mathbf{w} \in \Lambda\left(V_{\mathbb{Z}}\right)$. This argument appears in $[\mathrm{KM}]$ and, in a dual form, in KLW.

In general the desired lower bound is affected by the linear dependence relations between the components of $\tilde{\mathbf{f}}$. Namely, denote by $\mathcal{F}_{\mu, B}$ the $\mathbb{R}$-linear span of the restrictions of $1, f_{1}, \ldots, f_{n}$ to $B \cap \operatorname{supp} \mu$, denote its dimension by $s+1$, and choose 
functions $g_{1}, \ldots, g_{s}: B \cap \operatorname{supp} \mu \rightarrow \mathbb{R}$ such that $1, g_{1}, \ldots, g_{s}$ form a basis of $\mathcal{F}_{\mu, B}$. This choice defines a matrix

$$
R=\left(r_{i, j}\right)_{\substack{i=0, \ldots, s \\ j=0, \ldots, n}} \in M_{s+1, n+1}
$$

formed by coefficients in the expansion of $1, f_{1}, \ldots, f_{n}$ as linear combinations of $1, g_{1}, \ldots, g_{s}$. In other words, with the notation $\tilde{\mathbf{g}} \stackrel{\text { def }}{=}\left(1, g_{1}, \ldots, g_{s}\right)$, one has

$$
\tilde{\mathbf{f}}(x)=\tilde{\mathbf{g}}(x) R \quad \forall x \in B \cap \operatorname{supp} \mu .
$$

Moreover, since the first components of $\tilde{\mathbf{f}}$ and $\tilde{\mathbf{g}}$ are equal to 1 , the elements in the first column of $R$ are

$$
r_{i, 0}= \begin{cases}1 & \text { if } i=0, \\ 0 & \text { otherwise. }\end{cases}
$$

In view of $(4.7),\|\tilde{\mathbf{f}}(\cdot) \mathbf{c}(\mathbf{w})\|_{\mu, B}$ can be replaced by $\|\tilde{\mathbf{g}}(\cdot) R \mathbf{c}(\mathbf{w})\|_{\mu, B}$, and the latter, in view of linear independence of the components of $\tilde{\mathbf{g}}$, simply by the norms of vectors $R \mathbf{c}(\mathbf{w})$. Summarizing the discussion, we see that [3.2-ii] is equivalent to

$$
\begin{gathered}
\forall d>c \quad \exists T>0 \text { such that } \forall t \geq T, \forall j=1, \ldots, n \text { and } \forall \mathbf{w} \in \mathcal{S}_{n+1, j} \\
\text { one has } \max \left(e^{\frac{n+1-j}{n} t}\|R \mathbf{c}(\mathbf{w})\|, e^{-\frac{j}{n} t}\|\pi(\mathbf{w})\|\right) \geq e^{-j d t},
\end{gathered}
$$

where we have identified $\mathcal{S}_{n+1, j}$ with the set of elements of $\bigwedge^{j}\left(\mathbb{R}^{n+1}\right)$ representing $\Gamma \in \mathcal{S}_{n+1, j}$.

Here is another way to understand the above condition. Let $\mathbf{r}_{i}=\left(r_{i, 0}, \ldots, r_{i, n}\right)$ stand for the $i$ th row of $R, i=0, \ldots s$. Then, using (4.3), one can write the $i$ th component of $R \mathbf{c}(\mathbf{w})$ in the form

$$
\begin{aligned}
{[R \mathbf{c}(\mathbf{w})]_{i} } & =\sum_{k=0}^{n} r_{i, k} \sum_{J}\left\langle\mathbf{e}_{k} \wedge \mathbf{e}_{J}, \mathbf{w}\right\rangle \mathbf{e}_{J} \\
& =\sum_{J}\left\langle\sum_{k=0}^{n} r_{i, k} \mathbf{e}_{k} \wedge \mathbf{e}_{J}, \mathbf{w}\right\rangle \mathbf{e}_{J}=\sum_{J}\left\langle\mathbf{r}_{i} \wedge \mathbf{e}_{J}, \mathbf{w}\right\rangle \mathbf{e}_{J},
\end{aligned}
$$

and therefore, up to a uniform constant induced by replacing the Euclidean norm with the sup norm,

$$
\|R \mathbf{c}(\mathbf{w})\|=\max _{i=0, \ldots, s} \max _{\substack{J \subset\{1, \ldots, n\} \\ \# J=j-1}}\left|\left\langle\mathbf{r}_{i} \wedge \mathbf{e}_{J}, \mathbf{w}\right\rangle\right| .
$$

At this point it becomes useful to recall Lemma 3.1. Namely, for each $j=1, \ldots, n$ consider

$$
E=\left\{(\|R \mathbf{c}(\mathbf{w})\|,\|\pi(\mathbf{w})\|) \mid \mathbf{w} \in \mathcal{S}_{n+1, j}\right\} .
$$

It is clearly homogeneous with respect to positive integers, and the fact that it is discrete is easy from (4.11) and (4.8). Take $a=\frac{n+1-j}{n}$ and $b=\frac{j}{n}$, and recall that in the beginning we fixed $v \geq n$ and chose $c=\frac{v-n}{n(v+1)}$. Thus (4.9) amounts to saying that for any $c>c_{0} \stackrel{\text { def }}{=} j \frac{v-n}{n(v+1)}$ condition [3.1-ii] does not hold. By Lemma 3.1 , this is equivalent to saying that [3.1-i] does not hold with $v$ replaced by any real number greater than

$$
\frac{a+c_{0}}{b-c_{0}}=\frac{\frac{n+1-j}{n}+j \frac{v-n}{n(v+1)}}{\frac{j}{n}-j \frac{v-n}{n(v+1)}}=\frac{v+1-j}{j} .
$$


Therefore (4.9) becomes equivalent to

$$
\forall j=1, \ldots, n, \forall u>\frac{v+1-j}{j} \text { and } \forall \mathbf{w} \in \mathcal{S}_{n+1, j}
$$

with large enough $\|\pi(\mathbf{w})\|$, one has $\|R \mathbf{c}(\mathbf{w})\|>\|\pi(\mathbf{w})\|^{-u}$.

As a result, we managed to get rid of an auxiliary variable $t$ in (4.9) and found a way to directly involve $v$, rather than relate it to $c$ via (3.4).

Note that the only way the ball $B$, the measure $\mu$ and the map $\mathbf{f}$ enter the above conditions is via the matrix $R$, which depends on both $B \cap \operatorname{supp} \mu$ and $\mathbf{f}$ and is not uniquely determined - but another choice of $R$ would clearly yield a condition equivalent to $(4.9) \Leftrightarrow(4.12)$. Here is another useful way to describe $R$. Let

$$
\mathcal{L}=\langle\mathbf{f}(B \cap \operatorname{supp} \mu)\rangle_{a},
$$

put $s=\operatorname{dim}(\mathcal{L})$, and suppose

$$
\mathbf{h}: \mathbb{R}^{s} \rightarrow \mathcal{L} \text { is an affine isomorphism, and } \tilde{\mathbf{h}}(\mathbf{x})=\tilde{\mathbf{x}} R, \mathbf{x} \in \mathbb{R}^{s},
$$

where as usual we have $\tilde{\mathbf{h}} \stackrel{\text { def }}{=}\left(1, h_{1}, \ldots, h_{n}\right)$ and $\tilde{\mathbf{x}} \stackrel{\text { def }}{=}\left(1, x_{1}, \ldots, x_{s}\right)$. Then it is clear that $R$ and $\mathbf{g} \stackrel{\text { def }}{=} \mathbf{h}^{-1} \circ \mathbf{f}$ satisfy $(4.7)$, and that $1, g_{1}, \ldots, g_{s}$ generate $\mathcal{F}_{\mu, B}$ and are linearly independent over $\mathbb{R}$. This way, condition $(4.12) \Leftrightarrow(4.9) \Leftrightarrow[3.2$-ii] becomes a property of the 'enveloping subspace' $\langle\mathbf{f}(B \cap \operatorname{supp} \mu)\rangle_{a}$; in particular, $R$ can be chosen uniformly for all measures $\mu$, balls $B$ intersecting supp $\mu$ and maps $\mathbf{f}$ as long as (4.13) holds.

We are now ready for the main result of the section.

Theorem 4.2. Let $\mu$ be a Federer measure on a Besicovitch metric space $X, \mathcal{L}$ an affine subspace of $\mathbb{R}^{n}$, and let $\mathbf{f}: X \rightarrow \mathcal{L}$ be a continuous map such that $(\mathbf{f}, \mu)$ is good and nonplanar in $\mathcal{L}$. Then the following are equivalent for $v \geq n$ :

[4.2-i] $\left\{x \in \operatorname{supp} \mu \mid \mathbf{f}(x) \notin \mathcal{W}_{u}\right\}$ is nonempty for any $u>v$;

[4.2-ii] $\omega\left(\mathbf{f}_{*} \mu\right) \leq v$ (in other words, each of the sets in [4.2-i] has full measure);

[4.2-iii] (4.12) holds for some ( $\Leftrightarrow$ for any) $R$ satisfying (4.14).

This was proved in $\underline{\mathrm{K} 2}$ for $v=n$.

Proof. Obviously $[4.2-\mathrm{ii}] \Rightarrow[4.2-\mathrm{i}]$. Assuming [4.2-iii] and using the facts that $\mu$ is Federer and $(\mathbf{f}, \mu)$ is good, one can conclude that $\mu$-a.e. $x \in X$ has a neighborhood $V$ such that $\mu$ is $(C, \alpha)$-good and $D$-Federer on $V$ for some $C, D, \alpha>0$. Choose a ball $B=B(x, r)$ of positive measure such that the dilated ball $\tilde{B}=B\left(x, 3^{n+1} r\right)$ is contained in $V$, and note that (4.13) holds in view of (1.3). We have seen in (4.5) that for any $\mathbf{w}$, each of the coordinates of $g_{t} u_{\mathbf{f}} \mathbf{w}$ is expressed as a linear combination of functions $1, f_{1}, \ldots, f_{n}$. Therefore, in view of Lemma 1.1, [3.2-i] will hold (perhaps with a different constant $C$ ), and [3.2-ii] was postulated in the disguise of (4.12), as shown by the discussion preceding the statement of the theorem. Thus Proposition 3.2 applies, and [4.2-ii] follows.

On the other hand, saying that [4.2-iii] does not hold, in view of the same discussion, amounts to assuming that no ball $B$ intersecting supp $\mu$ satisfies $[3.2-$ ii]. From Lemma 4.1 it then follows that $\mathbf{f}(B \cap \operatorname{supp} \mu) \subset \mathcal{W}_{u}$ for some $u>v$, contradicting [4.2-i].

An especially remarkable feature of the above theorem is that whenever conditions [4.2-i,ii] hold for some $X, \mu$ and $\mathbf{f}$ satisfying the assumptions of the theorem, they hold for any $X, \mu, \mathbf{f}$ satisfying those assumptions. Indeed, condition [4.2-iii] 
equivalent to them has no reference to $\mathbf{f}, \mu$ or $X$, only to $\mathcal{L}$. In particular, one can make the most natural choice of $X, \mu, \mathbf{f}$; that is, put $X=\mathbb{R}^{s}, \mu=\lambda$ and $\mathbf{f}=\mathbf{h}$ as in (4.14), thus establishing Theorems 1.3 and 0.3 , and furthermore producing a formula for the Diophantine exponent of $\mathcal{L}$. Namely, one has

Corollary 4.3. Let $\mathcal{L}$ be an s-dimensional affine subspace of $\mathbb{R}^{n}$. Then

$$
\omega(\mathcal{L})=\max (n, \sup \{v \mid(4.12) \text { holds for } R \text { as in }(4.14)\}) .
$$

This will be made more explicit in the next section.

\section{Higher Diophantine EXponents And Theorem 0.4}

In this section we start by fixing a parametrization (0.6) for an $s$-dimensional affine subspace $\mathcal{L}$ of $\mathbb{R}^{n}$. This amounts to taking $R$ of the form

$$
R=R_{A} \stackrel{\text { def }}{=}\left(I_{s+1} \quad A\right)
$$

where $A \in M_{s+1, n-s}$. In order to restate condition (4.12) in terms of $A$, let us denote by $\mathbf{a}_{i}=\left(a_{i, s+1}, \ldots, a_{i, n}\right)$ the $i$ th row of $A, i=0, \ldots, s$, and identify it with an element of $V$ by putting $\mathbf{a}_{i}=\sum_{k=s+1}^{n} a_{i, k} \mathbf{e}_{k}$. Then, in view of (4.11), we have

$$
\left\|R_{A} \mathbf{c}(\mathbf{w})\right\|=\max _{i=0, \ldots, s} \max _{\substack{\mathcal{Z}\{1, \ldots, n\} \\ \# J=j-1}}\left|\left\langle\left(\mathbf{e}_{i}+\mathbf{a}_{i}\right) \wedge \mathbf{e}_{J}, \mathbf{w}\right\rangle\right| .
$$

Corollary 4.3 asserts that for $v \geq n$, the Diophantine exponent of $\mathcal{L}$ is not greater than $v$ if and only if for all $j=1, \ldots, n, u>\frac{v+1-j}{j}$ and $\mathbf{w} \in \mathcal{S}_{n+1, j}$ with large enough $\|\pi(\mathbf{w})\|$, the expression in (5.1) exceeds $\|\pi(\mathbf{w})\|^{-u}$.

This condition however can be considerably simplified. Namely, put

$$
V_{\bullet} \stackrel{\text { def }}{=} \mathbb{R} \mathbf{e}_{s+1} \oplus \cdots \oplus \mathbb{R} \mathbf{e}_{n},
$$

and denote by $\pi_{\bullet}$ the projection of $\bigwedge(V)$ to $\bigwedge\left(V_{\bullet}\right)$. We will also be using the following notation: $x \ll y$ will stand for $x<C y$, where $C$ depends only on the matrix $A$ and not on w.

Lemma 5.1. Suppose that $\left\|R_{A} \mathbf{c}(\mathbf{w})\right\|$ is less than 1 for some $\mathbf{w} \in \mathcal{S}_{n+1, j}$. Then $\|\mathbf{w}\| \ll 1+\left\|\pi_{\bullet}(\mathbf{w})\right\|$.

Proof. Let us take $I \subset\{0, \ldots, n\}$ of size $j$ and prove that the absolute value of $\left\langle\mathbf{e}_{I}, \mathbf{w}\right\rangle$ is bounded from above by a uniform constant times $\pi_{\bullet}(\mathbf{w})$. Denote by $k$ the smallest element of $I$. The claim is trivial if $k>s$. Otherwise, using (5.1), one can write

$$
\left|\left\langle\mathbf{e}_{I}, \mathbf{w}\right\rangle\right|<1+\left|\left\langle\mathbf{a}_{k} \wedge \mathbf{e}_{I \backslash\{k\}}, \mathbf{w}\right\rangle\right|
$$

and observe that, since $\mathbf{a}_{i} \subset V_{\bullet}$ for each $i$, the right hand side is not greater than $1+\max _{i}\left\|\mathbf{a}_{i}\right\| \cdot \max _{J \subset\{k+1, \ldots, n\}}\left|\left\langle\mathbf{e}_{J}, \mathbf{w}\right\rangle\right|$. The same argument can be applied to each of the components $\left\langle\mathbf{e}_{J}, \mathbf{w}\right\rangle$, and after no more than $s$ additional steps the process will terminate.

In particular, the lemma forces $\left\|R_{A} \mathbf{c}(\mathbf{w})\right\|$ to be not less than 1 for any $\mathbf{w} \in$ $\mathcal{S}_{n+1, j}$ with $j>n-s$ and large enough $\pi(\mathbf{w})$ (this was checked in $\mathrm{K} 2$, Lemmas 4.5 and 4.6] for some special cases). Since $1 \geq\|\pi(\mathbf{w})\|^{-u}$ whenever $u>\frac{v+1-j}{j}$ and $v$ is at least $j-1$, we can conclude that subgroups of rank greater than $n-s$ have no impact on the Diophantine exponent of $\mathcal{L}$. 
It will be convenient to associate to $A$ the following quantities: for each $j=$ $1, \ldots, n-s$, define

$$
\omega_{j}(A) \stackrel{\text { def }}{=} \sup \left\{v \mid \begin{array}{c}
\exists \mathbf{w} \in \mathcal{S}_{n+1, j} \text { with arbitrary large }\left\|\pi_{\bullet}(\mathbf{w})\right\| \\
\text { such that }\left\|R_{A} \mathbf{c}(\mathbf{w})\right\|<\left\|\pi_{\bullet}(\mathbf{w})\right\|^{-\frac{v+1-j}{j}}
\end{array}\right\} .
$$

By Lemma $5.1,(4.12)$ holds if and only if $\omega_{j}(A) \leq v$ for all $j=1, \ldots, n-s$. Thus Corollary 4.3 can be rewritten as

Corollary 5.2. For $\mathcal{L}$ parametrized by $(0.6), \omega(\mathcal{L})=\max \left(n, \omega_{j}(A)_{j=1, \ldots, n-s}\right)$.

We will refer to $\omega_{j}(A)$ as the Diophantine exponent of $A$ of order $j$. The reason for this terminology is the observation, essentially made in [K2], that

Lemma 5.3. $\omega_{1}(A)=\omega(A)$.

Proof. Take $\mathbf{v}=\left(\begin{array}{c}\mathbf{p} \\ \mathbf{q}\end{array}\right) \in \mathbb{Z}^{n+1} \backslash\{0\}=\mathcal{S}_{n+1,1}$ in place of $\mathbf{w}$, where $\mathbf{p} \in \mathbb{Z}^{s+1}$ and $\mathbf{q} \in \mathbb{Z}^{n-s}$, and observe that the only possible choice of $J$ in (5.1) and (4.3) is $J=\varnothing$. It follows that $\mathbf{c}(\mathbf{v})=\mathbf{v}, R_{A} \mathbf{c}(\mathbf{w})=\mathbf{p}+A \mathbf{q}$ and $\pi_{\bullet}(\mathbf{v})=\mathbf{q}$; hence the inequality in (5.2) coincides with (0.5).

In view of the lemma, the estimate $(0.7 \geq)$ stated in the Introduction gets to be a special case of Corollary 5.2 corresponding to $j=1$. However it is worthwhile to note that this inequality can be proved in an elementary way, and even more can be said:

Lemma 5.4. Let $\mathcal{L}$ be parametrized as in (0.6). Then for any $u<\omega(A)$ there exists an infinite subset $\mathcal{A}$ of $\mathbb{Z}^{n+1}$ such that

$$
|\mathbf{y q}+p|<\|\mathbf{q}\|^{-v} \text { for all } \mathbf{y} \in \mathcal{L} \text { and all but finitely many }(p, \mathbf{q}) \in \mathcal{A} \text {. }
$$

This not only proves the lower bound $(0.7 \geq)$, but also provides a way to approximate all points of $\mathcal{L}$ uniformly by a fixed sequence of integers.

Proof. One knows that for any $v<\omega(A)$ and infinitely many $\mathbf{q} \in \mathbb{Z}^{n-s}$ one can find $\mathbf{p}=\left(p_{0}, p_{1}, \ldots, p_{s}\right) \in \mathbb{Z}^{s+1}$ satisfying (0.5). Now take any $\mathbf{x} \in \mathbb{R}^{s}$, denote $\left(p_{1}, \ldots, p_{s}\right)$ by $\mathbf{p}^{\prime}$ and write

$$
\left|p_{0}+(\mathbf{x}, \tilde{\mathbf{x}} A)\left(\begin{array}{c}
\mathbf{p}^{\prime} \\
\mathbf{q}
\end{array}\right)\right|=\left|p_{0}+\mathbf{x p}^{\prime}+\tilde{\mathbf{x}} A \mathbf{q}\right|=|\tilde{\mathbf{x}}(A \mathbf{q}+\mathbf{p})| \leq\|\tilde{\mathbf{x}}\|\|A \mathbf{q}+\mathbf{p}\| .
$$

Slightly decreasing $v$ if needed, one gets

$$
\left|p_{0}+(\mathbf{x}, \tilde{\mathbf{x}} A)\left(\begin{array}{c}
\mathbf{p}^{\prime} \\
\mathbf{q}
\end{array}\right)\right| \leq\|\mathbf{q}\|^{-v}
$$

for all but finitely many $(\mathbf{p}, \mathbf{q})$ as above. Then it easily follows from (0.5) that $\|\mathbf{p}\|$ is bounded from above by $C\|\mathbf{q}\|$, where $C$ depends only on $A$. Thus, after possibly another slight change of $v$ and throwing away another finite subset, one can put $\left\|\left(\begin{array}{c}\mathbf{p}^{\prime} \\ \mathbf{q}\end{array}\right)\right\|^{-v}$ in the right hand side of (5.4).

We now turn to the equality cases in $(0.7 \geq)$, that is, to Theorem 0.4 . The first observation is that from Lemma 5.3 and Corollary 5.2 one can immediately deduce $(0.7=)$ for $s=n-1$, that is, for column matrices $\Leftrightarrow$ codimension one subspaces of 
$\mathbb{R}^{n}$. Since both $\omega(\mathcal{L})$ and $\omega(A)$ are obviously infinite if columns of $A$ are linearly dependent over $\mathbb{Q}$, this proves Theorem 0.4 in case (b).

Another case when Theorem 0.4 holds for trivial reasons is $\mathcal{L}=\{\mathbf{a}\}$, a zerodimensional subspace represented by a $1 \times n$ matrix $\mathbf{a} \in \mathbb{R}^{n}$. Indeed, it is a tautological statement that $\omega(\mathcal{L})$, that is, the Diophantine exponent of the $\delta$-measure supported at $\mathbf{a}$, is equal to $\omega(\mathbf{a})$. On the other hand everything done in $\S 4$ is easily applicable in the case $s=0$ (and $\tilde{\mathbf{x}}=1$ ). Thus it follows from Corollary 5.2 that $\omega_{j}(\mathbf{a}) \leq \omega(\mathbf{a})$ for each $j$. (Exercise: prove it directly from the definition (5.2).)

To finish the proof of Theorem 0.4 it remains to treat the case when $A$ is a matrix with rationally proportional rows. For that it will be useful to get a better understanding of the 'hidden symmetries' of higher order exponents. The three lemmas below serve this purpose.

Lemma 5.5. For any $A \in M_{s+1, n-s}$ and all $\mathbf{w} \in \mathcal{S}_{n+1, j}, 2 \leq j \leq n-s$, one has

$$
\max _{i=0, \ldots, s} \max _{\substack{J \subset\{0, \ldots, n\} \\ \# J=j-1}}\left|\left\langle\left(\mathbf{e}_{i}+\mathbf{a}_{i}\right) \wedge \mathbf{e}_{J}, \mathbf{w}\right\rangle\right| \ll\left\|R_{A} \mathbf{c}(\mathbf{w})\right\|
$$

and

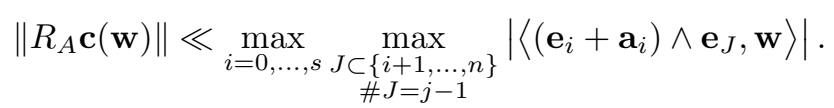

Note that in both cases the reverse inequalities are obvious. The statement is also obvious for $j=1$ (since, as was mentioned before, the only possible choice of $J$ is $J=\varnothing)$. Equation (5.5b) allows one to slightly reduce the set of pairs $(i, J)$ involved in computations of Diophantine exponents. Also, (5.5a) says that one can enlarge the set of pairs in (5.1) so that the formula, and hence the definition of $\omega_{j}(A)$, become symmetric under any permutation of rows of $A$.

Proof. A crucial observation is the following: for any $0 \leq k \leq s$, using (5.1) and the linearity of $\mathbf{u} \mapsto\left\langle\left(\mathbf{e}_{k}+\mathbf{a}_{k}\right) \wedge \mathbf{u}, \mathbf{w}\right\rangle$, one can write

$$
\left|\left\langle\left(\mathbf{e}_{k}+\mathbf{a}_{k}\right) \wedge \mathbf{u}, \mathbf{w}\right\rangle\right| \ll\left\|R_{A} \mathbf{c}(\mathbf{w})\right\|
$$

whenever $\mathbf{u} \in \bigwedge^{j-1}\left(V_{0}\right)$ has norm $\ll\|A\|$. Likewise, assuming in addition that $\mathbf{u} \in \bigwedge^{j-1}\left(\mathbb{R} \mathbf{e}_{k+1} \oplus \cdots \oplus \mathbb{R} \mathbf{e}_{n}\right)$, one has

$$
\left|\left\langle\left(\mathbf{e}_{k}+\mathbf{a}_{k}\right) \wedge \mathbf{u}, \mathbf{w}\right\rangle\right| \ll \max _{J \subset\{k+1, \ldots, n\}}\left|\left\langle\left(\mathbf{e}_{k}+\mathbf{a}_{k}\right) \wedge \mathbf{e}_{J}, \mathbf{w}\right\rangle\right| .
$$

To prove (5.5a), take $I \subset\{1, \ldots, n\}$ of cardinality $j-2$, and for any $0 \leq k \leq s$ write

$$
\begin{aligned}
\left|\left\langle\left(\mathbf{e}_{k}+\mathbf{a}_{k}\right) \wedge \mathbf{e}_{0} \wedge \mathbf{e}_{I}, \mathbf{w}\right\rangle\right| & =\left|\left\langle\mathbf{e}_{0} \wedge\left(\mathbf{e}_{k}+\mathbf{a}_{k}\right) \wedge \mathbf{e}_{I}, \mathbf{w}\right\rangle\right| \\
\text { (5.6a) with } \mathbf{u}=\left(\mathbf{e}_{k}+\mathbf{a}_{k}\right) \wedge \mathbf{e}_{I} & \left\|R_{A} \mathbf{c}(\mathbf{w})\right\|+\left|\left\langle\mathbf{a}_{0} \wedge\left(\mathbf{e}_{k}+\mathbf{a}_{k}\right) \wedge \mathbf{e}_{I}, \mathbf{w}\right\rangle\right| \\
& =\left\|R_{A} \mathbf{c}(\mathbf{w})\right\|+\left|\left\langle\left(\mathbf{e}_{k}+\mathbf{a}_{k}\right) \wedge \mathbf{a}_{0} \wedge \mathbf{e}_{I}, \mathbf{w}\right\rangle\right| \\
\text { (5.6a) with } \mathbf{u}=\mathbf{a}_{0} \wedge \mathbf{e}_{I} & \left\|R_{A} \mathbf{c}(\mathbf{w})\right\| .
\end{aligned}
$$

\footnotetext{
${ }^{2}$ The invariance of $\omega_{j}(A)$ under permutations not involving the top row is an immediate consequence of (5.1); however the fact that the top row can also be permuted is nontrivial.
} 
Similarly, if $k<i$ one has

$$
\begin{aligned}
& \left|\left\langle\left(\mathbf{e}_{i}+\mathbf{a}_{i}\right) \wedge \mathbf{e}_{k} \wedge \mathbf{e}_{I}, \mathbf{w}\right\rangle\right|=\left|\left\langle\mathbf{e}_{k} \wedge\left(\mathbf{e}_{i}+\mathbf{a}_{i}\right) \wedge \mathbf{e}_{I}, \mathbf{w}\right\rangle\right| \\
& \text { (5.6b) with } \mathbf{u}=\left(\mathbf{e}_{i}+\mathbf{a}_{i}\right) \wedge \mathbf{e}_{I} \quad \max _{J \subset\{k+1, \ldots, n\}}\left|\left\langle\left(\mathbf{e}_{i}+\mathbf{a}_{i}\right) \wedge \mathbf{e}_{J}, \mathbf{w}\right\rangle\right| \\
& +\left|\left\langle\mathbf{a}_{k} \wedge\left(\mathbf{e}_{i}+\mathbf{a}_{i}\right) \wedge \mathbf{e}_{I}, \mathbf{w}\right\rangle\right| \\
& \ll \max _{J \subset\{k+1, \ldots, n\}}\left|\left\langle\left(\mathbf{e}_{i}+\mathbf{a}_{i}\right) \wedge \mathbf{e}_{J}, \mathbf{w}\right\rangle\right|,
\end{aligned}
$$

and a repeated application of this trick allows one to reduce the estimation of $\left\|R_{A} \mathbf{c}(\mathbf{w})\right\|$ to $J \subset\{i+1, \ldots, n\}$, proving (5.5b).

The invariance of $\omega_{j}(A)$ with respect to permutations of rows of $A$ suggests that the same might hold for other row operations. This indeed happens to be the case:

Lemma 5.6. Let $A^{\prime}=B A$ for some $B \in \mathrm{GL}_{s+1}(\mathbb{Q})$; in other words, $A^{\prime}$ can be obtained from $A$ by a sequence of elementary row operations with rational coefficients (that is, transposition of rows, addition of one row to another, and multiplication of a row by a nonzero rational number). Then $\omega_{j}\left(A^{\prime}\right)=\omega_{j}(A)$ for all $j$.

Proof. Since row interchanges are taken care of in view of the previous lemma, it remains to prove

$$
\omega_{j}\left(A^{\prime}\right) \geq \omega_{j}(A)
$$

assuming all the rows of $A^{\prime}$ are the same as those of $A$ except for the top row, and the latter is equal to: (a) $\frac{k}{\ell} \mathbf{a}_{0}$, where $k$ and $\ell$ are nonzero integers, and (b) $\mathbf{a}_{0}+\mathbf{a}_{1}$.

For case (a), take $\mathbf{w} \in \mathcal{S}_{n+1, j}$, write it in the form $\mathbf{w}=\mathbf{w}_{0}+\mathbf{e}_{0} \wedge \mathbf{w}^{\prime}$ where both $\mathbf{w}_{0}$ and $\mathbf{w}^{\prime}$ are in $\Lambda\left(V_{0}\right)$, and put

$$
\tilde{\mathbf{w}}=\ell \mathbf{w}_{0}+k \mathbf{e}_{0} \wedge \mathbf{w}^{\prime} .
$$

It is easy to see that $\tilde{\mathbf{w}}$ also belongs to $\mathcal{S}_{n+1, j}$, that is, represents a subgroup of $\mathbb{Z}^{n+1}$ : one can write

$$
\mathbf{w}=\left(a \mathbf{e}_{0}+b \mathbf{v}_{1}\right) \wedge \mathbf{v}_{2} \wedge \cdots \wedge \mathbf{v}_{j}
$$

for some integer vectors $\mathbf{v}_{1}, \ldots, \mathbf{v}_{j} \in V_{0}$ and $a, b \in \mathbb{Z}$, and then take

$$
\tilde{\mathbf{w}}=\left(\ell a \mathbf{e}_{0}+k b \mathbf{v}_{1}\right) \wedge \mathbf{v}_{2} \wedge \cdots \wedge \mathbf{v}_{j} .
$$

Now we claim that $\left\|R_{A^{\prime}} \mathbf{c}(\tilde{\mathbf{w}})\right\|$ is not bigger than $\max (|k|,|\ell|)$ times $\left\|R_{A} \mathbf{c}(\mathbf{w})\right\|$. Indeed, one has, for $J \subset\{1, \ldots, n\}$,

$$
\begin{aligned}
\left\langle\left(\mathbf{e}_{0}+\frac{k}{\ell} \mathbf{a}_{0}\right) \wedge \mathbf{e}_{J}, \tilde{\mathbf{w}}\right\rangle & =\left\langle\mathbf{e}_{0} \wedge \mathbf{e}_{J}, k \mathbf{e}_{0} \wedge \mathbf{w}^{\prime}\right\rangle+\left\langle\frac{k}{\ell} \mathbf{a}_{0} \wedge \mathbf{e}_{J}, \ell \mathbf{w}_{0}\right\rangle \\
& =k\left(\left\langle\mathbf{e}_{J}, \mathbf{w}^{\prime}\right\rangle+\left\langle\mathbf{a}_{0} \wedge \mathbf{e}_{J}, \mathbf{w}_{0}\right\rangle\right)=k\left\langle\left(\mathbf{e}_{0}+\mathbf{a}_{0}\right) \wedge \mathbf{e}_{J}, \mathbf{w}\right\rangle,
\end{aligned}
$$

and also, for $i \geq 1$,

$\left\langle\left(\mathbf{e}_{i}+\mathbf{a}_{i}\right) \wedge \mathbf{e}_{J}, \tilde{\mathbf{w}}\right\rangle=\left\langle\left(\mathbf{e}_{i}+\mathbf{a}_{i}\right) \wedge \mathbf{e}_{J}, l \mathbf{w}_{0}\right\rangle=\ell\left\langle\left(\mathbf{e}_{i}+\mathbf{a}_{i}\right) \wedge \mathbf{e}_{J}, \mathbf{w}_{0}\right\rangle=\ell\left\langle\left(\mathbf{e}_{i}+\mathbf{a}_{i}\right) \wedge \mathbf{e}_{J}, \mathbf{w}\right\rangle$.

This clearly implies (5.7). In case (b) the argument is similar. Namely, we take $\mathbf{w} \in \mathcal{S}_{n+1, j}$, write it in the form

$$
\mathbf{w}=\mathbf{w}_{0}+\mathbf{e}_{0} \wedge \mathbf{w}_{0}^{\prime}+\mathbf{e}_{1} \wedge \mathbf{w}_{1}^{\prime}+\mathbf{e}_{0} \wedge \mathbf{e}_{1} \wedge \mathbf{w}^{\prime},
$$

where $\mathbf{w}_{0}, \mathbf{w}^{\prime}, \mathbf{w}_{1}^{\prime}, \mathbf{w}_{2}^{\prime}$ are all in $\Lambda\left(\mathbb{R} \mathbf{e}_{2} \oplus \cdots \oplus \mathbb{R} \mathbf{e}_{n}\right)$, and put

$$
\tilde{\mathbf{w}}=\mathbf{w}+\mathbf{e}_{0} \wedge \mathbf{w}_{1}^{\prime}=\mathbf{w}_{0}+\mathbf{e}_{0} \wedge\left(\mathbf{w}_{0}^{\prime}+\mathbf{w}_{1}^{\prime}\right)+\mathbf{e}_{1} \wedge \mathbf{w}_{1}^{\prime}+\mathbf{e}_{0} \wedge \mathbf{e}_{1} \wedge \mathbf{w}^{\prime} .
$$


Again, $\tilde{\mathbf{w}}$ can be easily shown to represent a subgroup of $\mathbb{Z}^{n+1}$ : write

$$
\mathbf{w}=\left(a \mathbf{e}_{0}+b \mathbf{e}_{1}+\mathbf{v}_{1}\right) \wedge\left(c \mathbf{e}_{1}+\mathbf{v}_{2}\right) \wedge \mathbf{v}_{3} \wedge \cdots \wedge \mathbf{v}_{j}
$$

for some integer vectors $\mathbf{v}_{1}, \ldots, \mathbf{v}_{j} \in \mathbb{R} \mathbf{e}_{2} \oplus \cdots \oplus \mathbb{R} \mathbf{e}_{n}$ and $a, b, c \in \mathbb{Z}$, and then take

$$
\tilde{\mathbf{w}}=\left(a \mathbf{e}_{0}+b\left(\mathbf{e}_{0}+\mathbf{e}_{1}\right)+\mathbf{v}_{1}\right) \wedge\left(c\left(\mathbf{e}_{0}+\mathbf{e}_{1}\right)+\mathbf{v}_{2}\right) \wedge \mathbf{v}_{3} \wedge \cdots \wedge \mathbf{v}_{j}
$$

Now let us estimate $\left\|R_{A^{\prime}} \mathbf{c}(\tilde{\mathbf{w}})\right\|$. It is clear that $\left\langle\left(\mathbf{e}_{i}+\mathbf{a}_{i}\right) \wedge \mathbf{e}_{J}, \tilde{\mathbf{w}}\right\rangle$ is the same as $\left\langle\left(\mathbf{e}_{i}+\mathbf{a}_{i}\right) \wedge \mathbf{e}_{J}, \mathbf{w}\right\rangle$ for $i \geq 1$ and $J \subset\{1, \ldots, n\}$. On the other hand,

$$
\begin{aligned}
\left\langle\left(\mathbf{e}_{0}+\mathbf{a}_{0}+\mathbf{a}_{1}\right) \wedge \mathbf{e}_{J}, \tilde{\mathbf{w}}\right\rangle & =\left\langle\mathbf{e}_{0} \wedge \mathbf{e}_{J}, \mathbf{w}+\mathbf{e}_{0} \wedge \mathbf{w}_{1}^{\prime}\right\rangle+\left\langle\mathbf{a}_{0} \wedge \mathbf{e}_{J}, \mathbf{w}\right\rangle+\left\langle\mathbf{a}_{1} \wedge \mathbf{e}_{J}, \mathbf{w}\right\rangle \\
& =\left\langle\left(\mathbf{e}_{0}+\mathbf{a}_{0}\right) \wedge \mathbf{e}_{J}, \mathbf{w}\right\rangle+\left\langle\mathbf{e}_{J}, \mathbf{w}_{1}^{\prime}\right\rangle+\left\langle\mathbf{a}_{1} \wedge \mathbf{e}_{J}, \mathbf{w}\right\rangle \\
& =\left\langle\left(\mathbf{e}_{0}+\mathbf{a}_{0}\right) \wedge \mathbf{e}_{J}, \mathbf{w}\right\rangle+\left\langle\left(\mathbf{e}_{1}+\mathbf{a}_{1}\right) \wedge \mathbf{e}_{J}, \mathbf{w}\right\rangle .
\end{aligned}
$$

Therefore $\left\|R_{A^{\prime}} \mathbf{c}(\tilde{\mathbf{w}})\right\| \leq 2\left\|R_{A} \mathbf{c}(\mathbf{w})\right\|$, so (5.7) holds as well.

Lemma 5.7. Suppose that $A$ has more than one row, and let $A^{\prime}$ be the matrix obtained from $A$ by removing one of its rows. Then $\omega_{j}\left(A^{\prime}\right) \geq \omega_{j}(A)$ for all $j$. If in addition the removed row is a rational linear combination of the remaining rows, then $\omega_{j}\left(A^{\prime}\right)=\omega_{j}(A)$ for all $j$.

Proof. Let $A^{\prime}$ be obtained from $A$ by removing its top row (this can be assumed without loss of generality in view of the row interchange invariance). It is clear that for any $\mathbf{w} \in \mathcal{S}_{n+1, j}$ the inner product $\left\langle\left(\mathbf{e}_{i}+\mathbf{a}_{i}\right) \wedge \mathbf{e}_{J}, \mathbf{w}\right\rangle$ coincides with $\left\langle\left(\mathbf{e}_{i}+\mathbf{a}_{i}\right) \wedge \mathbf{e}_{J}, \pi(\mathbf{w})\right\rangle$ whenever $i>0$ and $J \subset\{1, \ldots, n\}$. Thus it follows from (5.1) that $\left\|R_{A^{\prime}} \mathbf{c}(\pi(\mathbf{w}))\right\|$ is not greater than $\left\|R_{A} \mathbf{c}(\mathbf{w})\right\|$, and obviously $\pi(\mathbf{w}) \in \mathcal{S}_{n, j}$ and $\pi_{\bullet}(\pi(\mathbf{w}))=\pi_{\bullet}(\mathbf{w})$. Therefore whenever $\mathbf{w} \in \mathcal{S}_{n+1, j}$ produces 'a good approximation' to $A$ (meaning that $\left\|R_{A} \mathbf{c}(\mathbf{w})\right\|$ is smaller than $\left\|\pi_{\bullet}(\mathbf{w})\right\|$ to some negative power), its projection $\pi(\mathbf{w})$ onto $\bigwedge^{j}\left(V_{0}\right)$ yields an equally good (or better) approximation to $A^{\prime}$. This proves the first part of the lemma.

For the second part, in view of Lemma 5.6 it is enough to assume that all the coefficients in the linear combination are zero, that is, $\mathbf{a}_{0}=0$. Then one can reverse the above argument: whenever $\mathbf{w} \in \bigwedge^{j}\left(V_{0}\right)$ produces 'a good approximation' to $A^{\prime}$, it automatically yields an equally good approximation to $A$, since one has $\left\langle\left(\mathbf{e}_{0}+\mathbf{a}_{0}\right) \wedge \mathbf{e}_{J}, \mathbf{w}\right\rangle=\left\langle\mathbf{e}_{0} \wedge \mathbf{e}_{J}, \mathbf{w}\right\rangle=0$.

A combination of the above lemma with the observation made before Lemma 5.5 completes the proof of Theorem 0.4. In particular, we have shown that subspaces $\mathcal{L}$ of the form

$$
\mathcal{L}=\left\{\left(x_{1}, \ldots, x_{s}, a_{1}, \ldots, a_{n-s}\right)\right\} \quad \text { or } \quad \mathcal{L}=\left\{\left(x_{1}, \ldots, x_{s}, a_{1} x_{i}, \ldots, a_{n-s} x_{i}\right)\right\}
$$

satisfy $(0.7=)$, thus generalizing [K2, Lemma 4.7] (whose method of proof, borrowed from [BBKM], did not shed any light on higher Diophantine exponents of the corresponding matrices). Furthermore, in view of Lemma 5.4 one can conclude that whenever a subspace satisfying the assumptions of Theorem 0.4 is not extremal (equivalently, $\omega(A)>n$ ), for any $u<\omega(\mathcal{L})=\omega(A)$ one can find an infinite $\mathcal{A} \subset$ $\mathbb{Z}^{n+1}$ such that (5.3) holds; that is, there exists an infinite supply of approximating vectors which can work uniformly for all points of $\mathcal{L}$. 


\section{Generalizations and open questions}

6.1. For matrices with no rational dependence between rows or columns the exponents of orders higher than 1 seem to be hard to understand. In particular the following question, a special case of which was asked in [K2], appears to be interesting:

Question. Does there exist a matrix $A \in M_{s+1, n-s}$ and $2 \leq j \leq n-s$ such that $\omega_{j}(A)$ is greater than both $n$ and $\omega(A)$ ?

An affirmative answer to this question would give a counterexample to $(0.7=)$, and, moreover, would provide an example of a proper nonextremal affine subspace $\mathcal{L}$ of $\mathbb{R}^{n}$ such that for some $v<\omega(\mathcal{L})$ it is impossible to find an infinite subset $\mathcal{A}$ of $\mathbb{Z}^{n+1}$ such that $|\mathbf{y q}+p|<\|\mathbf{q}\|^{-v}$ for all $\mathbf{y} \in \mathcal{L}$ and all but finitely many $(p, \mathbf{q}) \in \mathcal{A}$. On the other hand, a useful consequence of the validity of $(0.7=)$ for all $\mathcal{L}$ would be a possibility to compute the Hausdorff dimension of the set of subspaces of a given dimension and Diophantine exponent: indeed, then, in view of [Do], one would have $(6.1)$

$$
\operatorname{dim}\left(\left\{\begin{array}{l|l}
\mathcal{L} \subset \mathbb{R}^{n} \mid \begin{array}{c}
\operatorname{dim}(\mathcal{L})=s \\
\omega(\mathcal{L})=v
\end{array}
\end{array}\right\}\right) \begin{cases}(s+1)(n-s-1)+\frac{n+1}{v+1} & \text { if } v>n \\
(s+1)(n-s) & \text { otherwise }\end{cases}
$$

By Theorem 0.4 this holds unconditionally for codimension one subspaces, that is,

$$
\operatorname{dim}\left(\left\{\begin{array}{l|c}
\mathcal{L} \subset \mathbb{R}^{n} & \begin{array}{c}
\operatorname{dim}(\mathcal{L})=n-1 \\
\omega(\mathcal{L})=v
\end{array}
\end{array}\right\}\right)= \begin{cases}\frac{n+1}{v+1} & \text { if } v>n \\
n & \text { otherwise }\end{cases}
$$

The author unfoundedly suspects (6.1) to be true regardless of the answer to the above question; in other words, even if higher order Diophantine exponents can interfere with $(0.7=)$, they conjecturally should not be powerful enough to affect the computation of the Hausdorff dimension.

6.2. The simplest matrices to look for possible counterexamples to $(0.7=)$ would be of size $2 \times 2$, corresponding to lines in $\mathbb{R}^{3}$. To convince the reader that the problem is far from trivial, let us work out an explicit formula for the second order Diophantine exponent of $A=\left(\begin{array}{l}\mathbf{a}_{0} \\ \mathbf{a}_{1}\end{array}\right)=\left(\begin{array}{ll}a_{02} & a_{03} \\ a_{12} & a_{13}\end{array}\right)$. Write $\mathbf{w} \in \mathcal{S}_{4,2}$ in the form

$$
\mathbf{w}=p \mathbf{e}_{0} \wedge \mathbf{e}_{1}+\sum_{\substack{i=0,1 \\ j=2,3}} w_{i j} \mathbf{e}_{i} \wedge \mathbf{e}_{j}+q \mathbf{e}_{2} \wedge \mathbf{e}_{3},
$$

and observe that for $i=0,1$ one has

$$
\begin{aligned}
& \left\langle\left(\mathbf{e}_{i}+\mathbf{a}_{i}\right) \wedge \mathbf{e}_{2}, \mathbf{w}\right\rangle=w_{i 2}-a_{i 3} q, \\
& \left\langle\left(\mathbf{e}_{i}+\mathbf{a}_{i}\right) \wedge \mathbf{e}_{3}, \mathbf{w}\right\rangle=w_{i 3}+a_{i 2} q,
\end{aligned}
$$

and also

$$
\begin{aligned}
\left\langle\left(\mathbf{e}_{0}+\mathbf{a}_{0}\right) \wedge \mathbf{e}_{1}, \mathbf{w}\right\rangle & =p-a_{02} w_{12}-a_{03} w_{13} \\
& =p-\operatorname{det}(A) q-a_{02}\left(w_{12}-a_{13} q\right)-a_{03}\left(w_{13}+a_{12} q\right) .
\end{aligned}
$$

Combining (6.3) and (6.4), one concludes that the definition (5.2) of $\omega_{2}(A)$ reduces to the following: $\omega_{2}(A)$ is the supremum of $v$ for which there exist $\mathbf{w} \in \mathcal{S}_{4,2}$ of the form (6.2) with arbitrary large $|q|$ such that

$\max \left(\left|w_{02}-a_{03} q\right|,\left|w_{12}-a_{13} q\right|,\left|w_{03}+a_{02} q\right|,\left|w_{13}+a_{12} q\right|,|p-\operatorname{det}(A) q|\right)<|q|^{-\frac{v-1}{2}}$. 
Even when $\operatorname{det}(A)=0$ (that is, rows/columns of $A$ are linearly dependent over $\mathbb{R}$ but not over $\mathbb{Q}$ ), the situation does not seem to be any less complicated.

6.3. Theorem 2.2 can be used to treat the so-called multiplicative versions of the Diophantine problems discussed in this paper. Namely, define

$$
\Pi_{+}(\mathbf{q}) \stackrel{\text { def }}{=} \prod_{q_{i} \neq 0}\left|q_{i}\right|
$$

denote by $\mathcal{W}_{v}^{\times}$the set of $\mathbf{y} \in \mathbb{R}^{n}$ for which there are infinitely many $\mathbf{q} \in \mathbb{Z}^{n}$ such that

$$
|\mathbf{y q}+p|<\Pi_{+}(\mathbf{q})^{-v / n}
$$

for some $p \in \mathbb{Z}$, and then define multiplicative Diophantine exponents:

- $\omega^{\times}(\mathbf{y})$ of $\mathbf{y}$ by $\omega^{\times}(\mathbf{y}) \stackrel{\text { def }}{=} \sup \left\{v \mid \mathbf{y} \in \mathcal{W}_{v}^{\times}\right\}$,

- $\omega^{\times}(\mu)$ of $\mu$ by $\omega^{\times}(\mu) \stackrel{\text { def }}{=} \sup \left\{v \mid \mu\left(\mathcal{W}_{v}^{\times}\right)>0\right\}$.

It is easy to see that $\omega^{\times}(\mathbf{y})$ is not less than $\omega(\mathbf{y})$ for all $\mathbf{y}$, and $\omega^{\times}(\mathbf{y})=n$ for $\lambda$-a.e. $\mathbf{y} \in \mathbb{R}^{n}$, that is, $\omega^{\times}(\lambda)=n$. To adapt the methods of the present paper to this set-up, one needs, following [KM] and [K2, §5], to replace the one-parameter flow (3.2) by the action of the multi-parameter semigroup

$$
\operatorname{diag}\left(e^{t}, e^{-t_{1}}, \ldots, e^{-t_{n}}\right), \quad \text { where } t_{i} \geq 0 \text { and } t=t_{1}+\cdots+t_{n} .
$$

This way it should be possible to prove Theorem 0.3 with $\omega$ replaced by $\omega^{\times}$(this was done in [K2] under the assumption that $\mathcal{L}$ is strongly extremal, that is, $\left.\omega^{\times}(\mathcal{L})=n\right)$, and also derive formulas for multiplicative Diophantine exponents of affine subspaces.

6.4. With some abuse of notation, let us denote by $\sigma(\mathbf{y})$ the Diophantine exponent of the column matrix given by the vector $\mathbf{y} \in \mathbb{R}^{n}$, that is,

$$
\sigma(\mathbf{y})=\sup \left\{v \mid \exists \infty \text {-many } q \in \mathbb{Z} \text { with }\|q \mathbf{y}+\mathbf{p}\|<|q|^{-v} \text { for some } \mathbf{p} \in \mathbb{Z}^{n}\right\},
$$

and, similarly to what was done with $\omega$, extend it to measures on $\mathbb{R}^{n}$. It follows from Khintchine's Transference Principle that $\omega(\mathbf{y})=n$ if and only iff $\sigma(\mathbf{y})$ attains its smallest possible value, i.e. is equal to $1 / n$. Therefore in all the problems related to the extremality of manifolds/measures, it makes no difference whether to interpret points of $\mathbb{R}^{n}$ as row vectors (linear forms) or as column vectors (the latter setup was employed in [KLW, KW1-2] and is usually referred to as simultaneous approximation, hence our choice of notation $\sigma$ ). The situation is however different when Diophantine exponents are bounded away from their critical values. Indeed, [C, Ch. V, Theorem IV] estimates $\sigma(\mathbf{y})$ in terms of $\omega(\mathbf{y})$ as follows:

$$
\frac{\omega(\mathbf{y})-n+1}{n} \geq \sigma(\mathbf{y}) \geq \frac{1}{n-1+n / \omega(\mathbf{y})}
$$

and the above inequalities are known to be sharp. Thus in general it is not possible to extract any information concerning $\sigma(\mu)$ when $\omega(\mu)$ is known.

On the other hand, the methods of this paper can be adapted to computations of the 'simultaneous Diophantine exponents' of manifolds and measures. Indeed, given a column vector $\mathbf{y} \in \mathbb{R}^{n}$ one simply needs to work with the collection of vectors of the form $\left(\begin{array}{c}q \mathbf{y}+\mathbf{p} \\ q\end{array}\right)$, where $\mathbf{p} \in \mathbb{Z}^{n}$ and $q \in \mathbb{Z}$, which is the same as $u_{\mathbf{y}} \mathbb{Z}^{n+1}$, 
where one uses $u_{\mathbf{y}} \stackrel{\text { def }}{=}\left(\begin{array}{cc}I_{n} & \mathbf{y} \\ 0 & 1\end{array}\right)$ instead of (3.1). Then, to study the simultaneous approximation properties of $\mathbf{y}$, one uses the action by

$$
g_{t}=\operatorname{diag}\left(e^{t / n}, \ldots, e^{t / n}, e^{-t}\right)
$$

which uniformly expands the first $n$ coordinates of vectors in $\mathbb{R}^{n+1}$ and contracts the last one. This way an application of Theorem 2.2 can yield Theorem 0.3 with $\omega$ replaced by $\sigma$, as well as, after a multi-parameter modification, by its multiplicative analogue.

6.5. Studying Diophantine exponents of matrices is a special case of a more general problem, where one replaces the right hand side of (0.5) by an arbitrary function of $\|\mathbf{q}\|$. Let us specialize to the case of row vectors and use the following definition [K2, §6.3]: for a nonincreasing function $\psi: \mathbb{N} \rightarrow(0, \infty)$, let $\mathcal{W}_{\psi}$ stand for the set of $\mathbf{y} \in \mathbb{R}^{n}$ for which there are infinitely many $\mathbf{q} \in \mathbb{Z}^{n}$ such that

$$
\|\mathbf{y q}+p\| \leq \psi(\|\mathbf{q}\|) \quad \text { for some } p \in \mathbb{Z} .
$$

By Groshev's Theorem (see e.g. [Sc2]) almost no (resp., almost all) y $\in \mathbb{R}^{n}$ belong to $\mathcal{W}_{\psi}$ if the series

$$
\sum_{k=1}^{\infty} k^{n-1} \psi(k)
$$

converges (resp., diverges). More generally, in $\mathrm{BD}$. submanifolds $\mathcal{M}$ of $\mathbb{R}^{n}$ are called of Groshev type for convergence (resp., divergence) if the convergence (resp., divergence) of (6.5) implies that almost no (resp., almost all) points of $\mathcal{M}$ are in $\mathcal{W}_{\psi}$. It is known [BKM], $\mathrm{Be},[\mathrm{BBKM}]$ that nondegenerate submanifolds $\mathcal{M}$ of $\mathbb{R}^{n}$ are of Groshev type for both convergence and divergence.

The situation is much less understood when $\mathcal{M}$ as above is replaced by a proper affine subspace $\mathcal{L}$ of $\mathbb{R}^{n}$. Clearly $\omega(\mathcal{L})$ must be equal to $n$ in order for $\mathcal{L}$ to be of Groshev type for convergence (since the choice $\psi(k)=k^{-v}$ for $v>n$ makes the series (6.1) converge) but the converse is not likely to be true. On the other hand, in the following two cases it has been proved that $\mathcal{L}$ parametrized as in $(0.6)$ is of Groshev type for convergence under the assumption that $\omega(A)$ is strictly less than $n$ : when $\mathcal{L}$ is a line passing through the origin BBDD, and when it is of codimension one [G1]. In the former case $\mathcal{L}$ was also proved to be of Groshev type for divergence. Note that both cases fall into the framework of Theorem 0.4, that is, admit a simple formula $(0.7=)$ for the Diophantine exponent of $\mathcal{L}$. In view of Corollary 5.2 it seems natural to make the following

Conjecture. Let $\mathcal{L}$ be parametrized by (0.6), and suppose that $\omega_{j}(A)$ is strictly less than $n$ for every $j$. Then $\mathcal{L}$ is of Groshev type for both convergence and divergence.

The author expects this conjecture, as well as the multiplicative analogue of its convergence case, to be provable by a combination of the methods of [BKM], [BBKM], G1], G3] and the present paper.

More generally, for any submanifold of $\mathbb{R}^{n}$ it should be possible to state its own version of Groshev's Theorem, with the convergence/divergence of (6.5) replaced 
by another 'dividing line' condition. The following problems, posed in [K2, still remain wide open:

- is it true that the aforementioned 'dividing line' condition of an affine subspace $\mathcal{L}$ of $\mathbb{R}^{n}$ is always inherited by manifolds nondegenerate in $\mathcal{L}$ ?

- given a general affine subspace, say parametrized as in (0.6), find its 'dividing line' condition, say in terms of the Diophantine properties of the parametrizing matrix $A$; or, vice versa, describe the class of subspaces with a given 'dividing line'.

6.6. Finally, we remark that the generality of Theorem 2.2 allows applications far beyond Diophantine approximation over $\mathbb{R}$. Namely, one can similarly consider Diophantine properties of measures on vector spaces over non-Archimedean local fields, both of characteristic zero $\mathrm{KT}$ and of positive characteristic G2. In fact, in the aforementioned two papers it was proved that manifolds nondegenerate in the ambient spaces are extremal (and moreover strongly extremal). An application of Theorem 2.2 can extend these results to manifolds nondegenerate in proper affine subspaces.

\section{ACKNOWLEDGEMENTS}

The author is thankful to Gregory Margulis, Barak Weiss and the reviewer for their valuable comments. This work was supported in part by NSF Grant DMS0239463.

\section{REFERENCES}

[Be] V. Beresnevich, A Groshev type theorem for convergence on manifolds, Acta Math. Hungar. 94 (2002), 99-130. MR1905790 (2003d:11109)

[BBDD] V. Beresnevich, V. Bernik, H. Dickinson, and M. M. Dodson, On linear manifolds for which the Khinchin approximation theorem holds, Vestsi Nats. Acad. Navuk Belarusi. Ser. Fiz.-Mat. Navuk (2000), 14-17 (Belorussian). MR1820985(2001j:11068)

[BBKM] V. Beresnevich, V. Bernik, D. Kleinbock, and G. A. Margulis, Metric Diophantine approximation: the Khintchine-Groshev theorem for non-degenerate manifolds, Moscow Math. J. 2 (2) (2002), 203-225. MR.1944505 (2004b:11107)

[BD] V. Bernik and M. M. Dodson, Metric Diophantine approximation on manifolds, Cambridge Univ. Press, Cambridge, 1999. MR.1727177 (2001h:11091)

[BKM] V. Bernik, D. Kleinbock, and G. A. Margulis, Khintchine-type theorems on manifolds: the convergence case for standard and multiplicative versions, Internat. Math. Res. Notices (9) (2001), 453-486. MR1829381 (2002g:11102)

[C] J.W.S. Cassels, An introduction to Diophantine approximation, Cambridge Tracts in Math., vol. 45, Cambridge Univ. Press, Cambridge, 1957. MR0087708 (19:396h)

[Da1] S. G. Dani, On invariant measures, minimal sets, and a lemma of Margulis, Invent. Math. (51) (1979), 239-260. MR530631 (80d:58039)

[Da2] On orbits of unipotent flows on homogeneous spaces, Ergod. Th. Dynam. Sys. (4) (1984), 25-34. MR758891 (86b:58068)

[Da3] - Divergent trajectories of flows on homogeneous spaces and Diophantine approximation, J. Reine Angew. Math. 359 (1985), 55-89. MR794799 (87g:58110a)

[Da4] On orbits of unipotent flows on homogeneous spaces, II, Ergod. Th. Dynam. Sys. (6) (1986), 167-182. MR857195 (88e:58052)

[Do] M. M. Dodson, Hausdorff dimension, lower order and Khintchine's theorem in metric Diophantine approximation, J. Reine Angew. Math. 432 (1992), 69-76. MR.1184759 (94a:11125)

[G1] A. Ghosh, A Khintchine-type theorem for hyperplanes, J. London Math. Soc. 72 (2) (2005), 293-304. MR2156655 (2006h:11089) 
[G2] - Metric Diophantine approximation over a local field of positive characteristic, J. Number Theory 124 (2) (2007), 454-469. MR2321374 (2008g:11120)

[G3] Dynamics on homogeneous spaces and Diophantine approximation on manifolds, Ph. D. Thesis, Brandeis University, 2006.

[K1] D. Kleinbock, Some applications of homogeneous dynamics to number theory, in: Smooth Ergodic Theory and Its Applications (Seattle, WA, 1999), Proc. Symp. Pure Math., vol. 68, Amer. Math. Soc., Providence, RI, 2001, pp. 639-660. MR 1858548 (2002g:37009)

[K2] Extremal subspaces and their submanifolds, Geom. Funct. Anal. 13 (2) (2003), 437-466. MR1982150 (2004f:11073)

[K3] — Baker-Sprindžuk conjectures for complex analytic manifolds, in: Algebraic groups and Arithmetic, TIFR, India, 2004, pp. 539-553.

[KLW] D. Kleinbock, E. Lindenstrauss, and B. Weiss, On fractal measures and Diophantine approximation, Selecta Math. 10 (4) (2004), 479-523. MR2134453 (2006g:11151)

$[\mathrm{KM}] \quad$ D. Kleinbock and G. A. Margulis, Flows on homogeneous spaces and Diophantine approximation on manifolds, Ann. Math. 148 (1998), 339-360. MR.1652916 (99j:11083)

$[\mathrm{KT}] \quad$ D. Kleinbock and G. Tomanov, Flows on S-arithmetic homogeneous spaces and applications to metric Diophantine approximation, Comm. Math. Helv. 82 (2007), 519-581. MR 2314053

[KW1] D. Kleinbock and B. Weiss, Badly approximable vectors on fractals, Israel J. Math. 149 (2005), 137-170. MR2191212

[KW2] , Friendly measures, homogeneous flows and singular vectors, in: Algebraic and Topological Dynamics, Contemp. Math., vol. 385, AMS, Providence, RI, 2005, pp. 281292. MR2180240 (2006f:11084)

[KW3] Dirichlet's theorem on diophantine approximation and homogeneous flows, J. Mod. Dyn. 2 (1) (2008), 43-62. MR2366229

[Mr1] G. A. Margulis, On the action of unipotent group in the space of lattices, Proceedings of the Summer School on group representations (Budapest 1971), Académiai Kiado, Budapest, 1975, pp. 365-370. MR0470140 (57:9907)

[Mr2] - Diophantine approximation, lattices and flows on homogeneous spaces, in: A panorama of number theory or the view from Baker's garden (Zürich, 1999), Cambridge Univ. Press, Cambridge, 2002, pp. 280-310. MR.1975458 (2004h:11031)

[Mt] P. Mattila, Geometry of sets and measures in Euclidean spaces. Fractals and rectifiability, Cambridge Studies in Advanced Mathematics, 44, Cambridge University Press, Cambridge, 1995. MR.1333890 (96h:28006)

[PV] A. Pollington and S. Velani, Metric Diophantine approximation and 'absolutely friendly' measures, Selecta Math. 11 (2) (2005), 297-307. MR2183850 (2006k:11142)

[Rg] M.S. Raghunathan, Discrete subgroups of Lie groups, Springer-Verlag, Berlin and New York, 1972. MR0507234(58:22394a)

[Rt1] M. Ratner, Raghunathan's topological conjecture and distributions of unipotent flows, Duke Math. J. 63 (1991), 235-280. MR.1106945 (93f:22012)

[Rt2] - Invariant measures and orbit closures for unipotent actions on homogeneous spaces, Geom. Funct. Anal. 4 (1994), 236-257. MR1262705 (95c:22018)

[Sc1] W. Schmidt, Diophantine approximation and certain sequences of lattices, Acta Arith. 18 (1971), 178-195. MR0286751 (44:3960)

[Sc2] , Diophantine approximation, Springer-Verlag, Berlin and New York, 1980. MR0568710 (81j:10038)

[Sp] V. Sprindžuk, Achievements and problems in Diophantine approximation theory, Russian Math. Surveys 35 (1980), 1-80. MR586190(81j:10039)

[SU] B. Stratmann and M. Urbański, Diophantine extremality of the Patterson measure, Math. Proc. Cambridge Phil. Soc. 140 (2006), no. 2, 297-304. MR2212281|(2007g:11090)

[U] M. Urbański, Diophantine approximation of self-conformal measures, J. Number Th. 110 (2005), 219-235. MR2122607(2006a:11100)

Department of Mathematics, Brandeis University, Waltham, Massachusetts 024549110

E-mail address: kleinboc@brandeis.edu 